\title{
Die Hysterie Neurose oder Psychose?
}

\author{
Von \\ Dr. Ernst Herzig, \\ Wien-Steinhof. \\ (Eingegangen am 7. Juni 1917.)
}

Der Verfolg der Literatur, die im Laufe der Jahrhunderte über Hysterie geschrieben wurde, läßt ein zu allen Zeiten als typisch hervorgehobenes Symptom im Krankheitsbilde hervortreten: Die Auslösung und Veränderbarkeit körperlicher, unter Berücksichtigung des Menschentypus als krankhaft zu bezeichnender Erscheinungen durch geistige Prozesse. Durch die letzten drei Worte hatten die älteren Autoren diese Krankheit gegen die Epilepsie abgegrenzt, welche als ein rein nervöser Proze $B$ aufgefaßt wurde und als dasjenige, was heute als Reflexneurose bezeichnet wird. $\mathrm{Zu}$ jenen Zeiten hatte man den Begriff der Hysterie in den Krampfanfällen erschöpft, da die experimentelle Untersuchung nicht weiter vorzudringen vermochte und man die heute stark betonten Stimmungs- und Charakteranomalien als typisch hysterische zu betonen und von ähnlichen Schwankungen bei anderen Krankheitsbildern $\mathrm{zu}$ trennen, weil ihnen in diesem Punkte differentialdiagnostische Momente fehlten, keinen Anhaltspunkt zur Hand hatte. Während man damals alle jene eben genannten Anomalien einfach unbeachtet ließ, sobald die Behandlung der Hysteriefrage als Thema aufgeworfen wurde, glaubt heute mancher, ohne weitere Unterstützung dieselben auf den Anblick hin als hysterische klassifizieren respektive von dieser Klassifikation ausschließen zu können. Der Fehler liegt beim Beobachter, der die ganze Geistesarbeit, welche ihn nach wenn auch rascher Kenntnisnahme aller aus der Beobachtung sicher hysterischer Fälle bekannten hysterischen Eigentümlichkeiten einen Wahrscheinlichkeitsschluß für den gegebenen Fall zu machen, in den Stand setzt, ignoriert. Das Gefühl eines Gewißheitsschlusses ist in einem solchen Falle auch in seiner Verursachung rein subjektive Beigabe.

Welche körperlichen Symptome als hysterische zu betrachten seien, darauf führt die alte Auffassung hin, welche Krampfanfälle bestimmter Form als das Charakteristicum der Krankheit Hysterie ansah. Sie brachte damit zum Ausdrucke, daß in dem Auftreten jener Krämpfe ein Abweichen vom allgemeinen Reaktionstypus liege, der dasselbe als ein 
krankhaftes kennzeichne. Dieses vom Typus Abweichende macht sie als etwas Abnormes kenntlich, als etwas, was zu dem voraufgehenden psychischen Prozesse die natürliche Äquivalenz vermissen läßt.

Die Ausdrücke Hysterie und hysterisch hat man im Ablaufe der Zeiten recht unbestimmt werden lassen, seitdem man die Hysterie mit der Hypochondrie, der Epilepsie und mit der Neurasthenie zusammenwarf. In den letzten abgelaufenen drei Jahrzehnten ist man fast einstimmig dazu gekommen, die Neurose Hysterie von den anderen Neurosen abzutrennen, zum großen Teile auf Grund der lebhaften Diskussionen, welche in dieser Zeit über ihre Abgrenzung geführt wurden. Viel Wirrwarr in der richtigen Deutung und Verwertung dieser Errungenschaft richtete die Lehre der Salpêtrière an. Mit ihrer Behauptung der psychotischen Natur der Hysterie hat sie in vielen Köpfen den eben erst errungenen Erfolg, die nosologische Einheit der Hysterie erkannt zu haben, durch ihren Irrtum quitt gemacht. Derselbe ist auch heute noch in den Kreisen vieler Psychopathologen lebendig. Daher mag es kommen, daß man vielfach das vorzüglichste Erkennungsmerkmal der Hysterie in den psychischen Eigenheiten der Hysteriker fand, während man die körperlichen Krankheitszeichen in den Hintergrund schieben zu können vorgab. Ein Selbstbetrug, indem gerade Psychopathologen dieser Richtung darauf zittern, endlich einmal durch Beobachtung eines hysterischen Anfalles eine Bestätigung ihrer Diagnose zu erhalten. Die Mehrdeutigkeit der bei den Hysterikern vorkommenden geistigen Eigentümlichkeiten gestattet nämlich keine Abgrenzung gegenüber den anderen in Betracht kommenden Neurosen und gegebenenfalls Psychosen. Ebensowenig wie jene Eigentümlichkeiten an sich, geben deren Intensität und Form bestimmte Maße an die Hand, aus denen auf ihre Grundlegung ein auch nur halbwegs richtiger Schluß gezogen werden könnte. Daß eine aus den psychischen Symptomen allein ohne von früher her erworbene Kenntnis einer Person auf Hysterie lautende Diagnose schließlich wirklich in ihrer Richtigkeit bestätigt wurde, gebe ich ohne weiteres $\mathrm{zu}$, frage aber: wodurch erfolgte jene Bestätigung? Durch Nachweis der körperlichen Stigmata. Damit wird nicht geleugnet, daß jene geistigen Symptome in gleicher Weise wie die körperlichen tatsächliche Hervorbringungen der Krankheit Hysterie sind, respektive sein können.

Die Auffassung der Hysterie als Psychose bringt keine Erklärung, weil sie eine unbekannte Größe durch eine andere ebenso unbekannte erläutern will. Alle auf psychischem Wege entstandenen Krankheitserscheinungen als hysterische zu erklären, widerspricht der Erfahrung, welche z. B. die Angstneurosen und die nervösen Insuffizienzzustände als nur psychisch bedingte kennt, welche aber als hysterische Krankheitserscheinungen zu bezeichnen, niemandem in den Sinn kommt. Selbst 
dann, wenn dieselben bei Hysterikern auftreten, trägt man Bedenken, sie in ihrer Ätiologie mit anderen zu identifizieren, welche auf die Hysterie als ihre Grundlegung zurückzuführen sind. Mehr als alle anderen Hysterietheorien scheint mir gerade die in Frage stehende Theorie darunter zu leiden, daß sie nicht nach vorausgegangener $\mathrm{Zu}$ sammenfassung alles dessen, was man als hysterisch bezeichnet, eine für alle diese Erscheinungen zutreffende Erklärung sucht, sondern in der überströmenden Freude darüber, in einer großen Zahl von sicher hysterischen Krankheitsfällen die gewiß zutreffende Erklärung gefunden zu haben, dieselbe nun auf alle Fälle überträgt. Jene große Zahl sind: die Hysteriefälle durch Vorstellung ausgelöst. Wenn man also ,die $\mathrm{Hy}_{\text {* }}$ sterie eine Psychose" als die bedeutendste Errungenschaft der Hysterieforschung hinstellt, so übersehe man nicht, daß damit dem Belieben der einzelnen Autoren weiter Spielraum gelassen wird. Je nach der Definition wird man die Psychosen in weiterem und engerem Rahmen abgrenzen können. Schließlich könnte man auch darauf hinweisen, daß zwischen Geistes- und Nervenkrankheiten kein prinzipieller Unterschied zu machen sei, da Krankheiten der Seele Gehirnkrankheiten seien.

Damit berühre ich einen Hauptpunkt der Hysteriefrage. Man hat die Hysterie von den anderen Neurosen abgetrennt, man hat sie als eine einheitliche Krankheit erkannt, man hat sie bald als Krankheit im eigentlichen Sinne, bald als krankhafte Reaktionsweise bezeichnet. Und das Charakteristicum der Hysterie? Die allgemeine Unklarheit über den Begriff bekundet sich in der Verlegenheit der Antworten, die sich mit gewissen und eigenartigen Störungen der Motilität und Sensibilität und eigenartiger seelischer Reaktionsweise über die Schwierigkeit derselben hinwegzuhelfen suchen. Nach den Ausführungen des vorhergehenden Absatzes brauche ich mich nur mit den körperlichen Eigentümlichkeiten näher zu befassen. Die Vollständigkeit der Aufzählung aller hierher gehörigen Stigmata in den verschiedenen Monographien der Hysterie und den Arbeiten über dieselbe überhebt mich jeder Wiederholung. Keines dieser Stigmata, an sich betrachtet in seinem Auftreten als krankhafte Erscheinung, ist für Hysterie charakteristisch. Charakteristisch ist die Art ihres Auftretens. Zu den häufigsten Symptomen gehören die Contracturen und die klonischen Krämpfe, die Muskellähmungen, die Stimmbandlähmungen, die Hyperalgesie und die Analgesie. An sich sind diese Erscheinungen keine Symptome der Hysterie; denn sie können auch innerhalb einer Hysterie organisch, nicht nur funktionell bedingt vorkommen. Der Nachweis des funktionellen Ursprunges besagt zugleich, daß die grundlegende Krankheit die Hysterie sei; der Entstehungsmechanismus ist entscheidend für die Diagnose. 
Dieser Entstehungsmechanismus ist charakterisiert durch ein aktives Schaffen der Hysterie. Denn das Charakteristische derselben ist ihre Fähigkeit, Krankheitssymptome wirklich zu erzeugen, etwas an den Tag zu bringen, was vorher nicht da war. Semi Meyer hat vorgeschlagen, diese Eigenschaft in Anlehnung an das sonstige Leben als Produktivität zu bezeichnen und die Krankheitserscheinungen als Produkte. Dieser Ausdruck gibt die spezifische Eigenheit der Hysterie an und grenzt sie dadurch gleichzeitig von allen anderen funktionellen Neurosen ab. Uber die Genese macht derselbe keine Andeutung. Jede Theorie der Hysterie kann sich ihre volle Selbständigkeit wahren.

In manchen Psychopathologen sitzt die Uberzeugung fest, daß es sich bei den hysterischen Symptomen um keine echten und wahren Krankheitserscheinungen handle; sie haben sich gewöhnt, Hysterikern, wenn sie dieselben schon nicht als Simulanten reinsten Wassers bezeichnen, ihren Platz in unmittelbarster Nähe derselben zu bestimmen. Der Schmerz, den die Hysteriker zu haben vorgeben, soll kein Schmerz sein; was man an Contracturen und Lähmungen zu sehen bekommt, soll nur durch eine dauernde Willensverkehrtheit zustande kommen. Man vergißt dabei, daß auch die Simulation eine adäquate Motivierung verlangt. Ist schon der Gedanke an eine so weitverbreitete und weitgehende Simulationstendenz, wie sie nach dieser Auffassung in der Hysterie sich ausdrücken würde, für eine wissenschaftliche Auffassung, insbesondere für eine teleologische Erfassung des Lebens, unfaßbar, so macht schon gar der Motivationsmangel ihn psychologisch unhaltbar. In treffender Weise wurden die von den Simulanten vorgetäuschten Symptome als Produktionen gegenüber den Produkten der Hysterie bezeichnet.

Es können übrigens auch körperliche Erscheinungen als Hervorbringungen der Hysterie auftreten, welche man nicht zu den Krankheitserscheinungen rechnen kann. So soll (ich weiß darüber nur aus der Literatur, während in der eigenen Erfahrung ein solcher Fall mir noch nicht unterkam) das Auftreten von Schwangerschaftszeichen in Form zunehmenden Volumens des Abdomens ein nicht selten zu beobachtendes Symptom der Hysterie sein. Das würde eben nur darauf hinweisen, daß überhaupt körperliche Erscheinungen durch die erwähnte Krankheit ausgelöst werden können. Solange dieselben aber nicht durch ihre Krankhaftigkeit die Aufmerksamkeit des Beobachtenden in besonderer Weise erregen und zu therapeutischen Maßnahmen auffordern, kann deren Nichtbeachtung ohne Schaden geschehen. Sie können allerdings zu einer Erkenntnis der psychopathischen Persönlichkeit führen unter Umständen zu einem Zeitpunkte, wo das Fehlen von Krankheitserscheinungen diese Erkenntnis noch nicht ermöglicht. Diese rein theoretische Erwägung dürfte kaum jemals in Betracht kommen, wenn nicht 
vielleicht das genannte Auftreten von Schwangerschaftszeichen als erstes und vonderhand einziges Hysteriesymptom vorhanden ist.

Dieser Meinung dürfte die D ubois' scharf gegenüberstehen, der als das einzige wirkliche Charakeristicum der Hysterie die konvulsiven Anfälle ansieht, während er von allen anderen Zufällen behauptet, daß sie zwar auch auftreten könnten, daß man sie aber nicht auf die Hysterie beziehen müsse.

Die Hysterie bezeichne ich also als eine funktionelle Neurose, welche durch die Fähigkeit, wirkliche Krankheitserscheinungen entstehen zu lassen, charakterisiert ist.

Krankhaft wird ein körperlicher Vorgang (hier selbstverständlich im Sinne der einem affektiven Vorgang entsprechenden Ausdrucksbewegung) dadurch, daß er in seiner Qualität, Intensität oder Extensität von jenem abweicht, welcher nach der Norm mit einem bestimmten Affekte verbunden ist. Diese Abweichung findet ihren psychologischen Ausdruck in dem Fehlen der dem körperlichen Vorgange zugrunde liegenden Motivation, im Fehlen jeder regelmäßigen Koordination, welche zwischen Affekt und Ausdrucksbewegung samt den ihr vorausgehenden Innervationen besteht und vermöge welcher wir -- nach W undt - jede Ausdrucksbewegung als ein adäquates Symptom der entsprechenden seelischen Regung betrachten lernen. In der Psychopathologie hat man seit langem die wichtige Tatsache aus dem Gebiete der Psychophysik des Gefühlslebens verzeichnet, daß es eine Gruppe geistiger Erkrankungen gibt, in der jene die normale Psychognostik leitende Beziehung eine Änderung erfahren hat. Die Beobachtung führte zur Erkenntnis, daß bei manchen geistigen Erkrankungen zwischen dem wirklichen seelischen Erlebnis mit seinem Affekte und dem psychischen Ausdruck des letzteren eine Kluft bestehe, so daß der Beobachter aus dem Ausdrucke der äußeren Erscheinungen auf einen ganz anderen Gefühlszustand verwiesen wird, als derselben nach den in der normalen Psychologie gesammelten Kenntnissen dem Gegenstande entsprechen sollte. Neben der Dementia praecox erkannte man schon frühzeitig an der Hysterie ein Feld, auf welchem jene gesetzmäßige Zuordnung zu fehlen schien. Dieses Zusammentreffen dürfte ein Grund sein, warum so oft eine beginnende Dementia praecox von einer Hysterie nicht mit wünschenswerter Schärfe abgegrenzt werden kann. Findet eine solche Abweichung nur in der Intensität oder Extensität statt, dann hat man damit noch keinen Vorgang vor sich, welcher jenes Merkmal an sich hätte, das denselben als hysterischen charakterisieren würde. Ein solches ist erst dann gegeben, wenn dessen qualitative Neuheit gegenüber der Norm zu Erscheinung kommt.

Diese Definition legt das Wesen der Hysterie nicht einfach in eine abnorme Reizbarkeit des gesamten Nervensystems, infolge deren die 
verschiedenen Provinzen dieses Systems gegen die unbedeutendsten dynamischen, organischen und psychischen Einflüsse, welche andere gesunde Menschen gar nicht affizieren, eine exzessive Reaktion zeigen. Eine solche Reizbarkeit ist ja kein Spezificum der Hysterie, kommt in sehr ausgeprägter Form z. B. bei Neurasthenie vor. Das Abnorme in der Qualität bildet das verlangte Spezificum, die Fähigkeit, mit Krankheitserscheinungen auf Reize zu antworten.

Es existiert also ein prinzipieller Unterschied zwischen den Krankheitsgruppen Hysterie und Neurasthenie. Die Ansicht Ste yerthals unterschreibe ich nicht, daß die Hysterie nur einen höheren Grad der Neurasthenie darstelle. Während letztere nur eine intensive und extensive Abnormität gegenüber dem Typus bildet, ist die qualitative Abnormität das spezifizierende Kennzeichen der ersteren. Nur für letztere kann man das hemmungslose Zum-Ausdruck-Gelangen der übertriebenen physiologischen Ausdruckshandlungen auf Vorstellungen und Empfindungen hin als Merkmal ansehen, während für Hysterie gerade das Hinausfallen aus dem Umfange des Physiologischen das diagnostisch Maßgebende ist.

Auf eine solche Vermengung läuft auch die Ansicht Vogts hinaus, daB ,alle psychopathologischen Erscheinungen, welche uns im Krankheitsbilde der Hysterie entgegentreten, nur Intensitätsveränderungen normaler Phänomene sind".

Welche psychischen Prozesse kommen nun als auslösende in Betracht? Die Psyche besitzt drei Vermögen: Denken, Fühlen, Wollen. Das letztere ist der Urgrund aller menschlichen Äußerungen, seien dieselben normaler oder krankhafter Art. Die ersteren aber geben die inhaltliche Fundierung und das Fühlen macht die Differenz in den Formen, wie die einzelnen Individuen auf die Produkte des Erkenntnisvermögens reagieren. Während dieses letztere als ein seiner Natur nach rein passives Vermögen sich darstellt, erscheint das Gefühlsvermögen als ein in weiten Grenzen durch sich selbst veränderliches. Da auch die Willenskraft bei verschiedenen Individuen eine verschiedene ist, so ergibt sich für Abnormität in den Willenshandlungen eine zweifache Möglichkeit: entweder wird der Willenseffekt herbeigeführt durch die objektiv abnorme Gefühlsbetonung eines Erkenntnisaktes oder durch die Schwäche des Willens. Im ersten Falle kann sowohl die große Heftigkeit jede Gegenarbeit des Willens unterdrücken, wie andererseits die zu geringe Lebhaftigkeit den nötigen Willensanreiz fehlen lassen.

Erst ganz kürzlich ist der Vorschlag gemacht worden ${ }^{1}$ ), den ,,sinnlosen" Namen Hysterie durch ein bezeichnenderes Wort, etwa Thymekstasie, zu ersetzen. Die Autoren erhoffen sich von einem solchen Er-

$\left.{ }^{1}\right)$ Rieder und Leeser, Über die Beurteilung und neuere Behandlung der psychomotorischen Störungen. Zeitschr. f. d. ges. Neurol. u. Psych. 35, $425 \mathrm{ff}$. 
satze, daß damit vielem Streite der Boden entzogen würde. Indessen ersehe ich mir gar keinen Vorteil, weil dieses Wort das eben Gekennzeichnete, für die Hysterie Charakteristische, nicht zum Ausdruck bringt, wogegen das Wort Hysterie außer der Indifferenz über das bezüglich der Krankheitserscheinungen zu Sagende das Vorrecht einer eingesessenen Popularität für sich hat. Die im Worte Thymekstasie liegende Anlehnung an die platonische Dreiteilung der menschlichen Seele dürfte zu einer rascheren Einbürgerung kaum etwas beizutragen imstande sein.

„Ist eine nicht schnell vorübergehende Störung des gewöhnlichen psychophysischen Zusammenhanges entstanden, die wir als eine Krankheit auffassen, so ist für ihre Bezeichnung als Hysterie zunächst maßgebend, daß die Störung in erster Linie das Affektleben in folgender Weise betrifft: Die Einzelgefühle bekommen einen erhöhten Einflu $B$ auf die psychophysischen Lebensäußerungen, sie werden hemmungsloser und sind schnellem Wechsel unterworfen. Sie, neigen also zu unberechenbaren, plötzlichen und hochgradigen Schwankungen und, ihrer Natur nach im psychischen Leben die einzigen Vorgänge, die sich zwischen konträren Gegensätzen bewegen, bedingen sie, indem sie überwertig werden, den wandelbaren Charakter aller Krankheitserscheinungen.

Die Apperzeption bekommt dadurch, daß auch sie erhöht von Gefühlen geleitet ist, eine starre Richtung auf das Subjekt.

Bei den Willensvorgängen tritt der zielstrebige, verstandgeleitete Anteil zugunsten des affektiven zurück. Der Mangel an zielbewuBtem Wollen imponiert als Willenschwäche und Haltlosigkeit, die sich also mit den gelegentlichen explosiven Willensäußerungen der Hysterischen sehr wohl verträgt.

Diese Umänderung der psychischen GesetzmäBigkeit hat ihr Abbild oder ihre Wirkung (die Wahl des Ausdruckes hängt davon ab, ob man auf dem Boden des psychophysischen Parallelismus oder der Wechselwirkungstheorie steht) in den körperliehen Äußerungen der Hysterie. Die hemmungslosen Affektschwankungen erscheinen als übertriebene Ausdrucksbewegungen (bzw. als ihr völliges Versagen), fixiert durch die starre Richtung der Apperzeption und die Schwäche der Impulse zur Wiederherstellung der Ordnung. Von den theatralischen Schmerzensäu Berungen bis zu den paradox anmutenden visceralen Störungen haben wir, was die Deutung aus den genannten psychischen Umänderungen betrifft, in den Ausdrucksbewegungen ein vollständiges Analogon. So sind die sensorischen und sensiblen Ausfallsund Reizerscheinungen Äußerungen der eigenartigen Apperzeptionsrichtung."

Diese Ansicht erklärt ausdrücklichst, daß die starken Schwankungen in den psychophysischen Lebensäußerungen, welche durch die Eigenart des hysterischen Affektlebens verursacht werden, das Bild der Hysterie zusammensetzen, andererseits aber Äußerungen der eigenartigen Apperzeptionsrichtung seien. Hier ist es von Interesse, darauf hinzuweisen, daß mit dieser Auffassung eine Abgrenzung der Hysterie und auch der hysterischen Psychosen gegenüber anderen neuropathologischen und psychopathologischen Abnormitäten und Krankheiten nicht möglich ist; denn bei allen Formen der Degeneration und des degenerativen Irreseins treten solche Schwankungen auf. Was die Eigentümlichkeiten der Affektäußerungen anbelangt, kann man eine charakteristische 
Eigentümlichkeit der hysterischen Affektäußerungen gegenüber jenen bei den erwähnten Degenerationen nicht nennen. Die von den Autoren gegebene Schilderung beinhaltet nach meiner Utberzeugung eher eine psychologische Erfassung der Dementia praecox.

Die verschiedenen Theorien einer Psychopathologie der Hysterie gehören entweder der einen Richtung, welche rein psychisch diese Krankheit erklären will, an oder sie nehmen auch oder allein eine psychophysische Grundlage in die Ätiologie hinein. Zu den erstgenannten gehören die Theorien der großen französischen Hysterieforscher, welche auch die deutschen Psychiater zum größten Teile gefangenhält, welchem nur ein kleinerer als Anhängerschaft der zweiten Richtung gegenübersteht. Jene Unterschiede, wie jene psychische Grundeigenschaft zu benennen sei oder welche überhaupt in Betracht kommen könne, betreffen nicht das Wesen der zugegebenen Lösung. Man könnte diesen Theorien entgegenhalten, daß eine solche psychische unüberwindliche Schwäche nur aus einem psychophysischen Mißverhältnisse, aus einer psychophysischen Disproportion, ihre Erklärung finde, da die Natur der Psyche eine Erklärung aus sich heraus als eben ihrer Natur an sich widerstreitend nicht zuläßt.

Charcot gilt heute allgomein als derjenige Forscher, welcher die Hysteric zur Psychose stempelte, wenn auch nicht geleugnet werden soll, daß er damit zum Teile nur von seinem Lehrer Briquet übernommene Gedanken zum Ausdrucke brachte. Jedenfalls war or derjenige, der dem Gedanken in schärfster Form und klarster Weise die Bahn gebrochen hat.

Dieser Gedanke liegt im Satze: Hysterisch sind körperliche Vorgänge außergewöhnlicher Stärke und Art, welche durch Vorstellungen erzeugt werden. Dic rein körperlichen Begleiterscheinungen der Hysterie hat Char cot nicht übersehen, aber es kam ihm nie in den Sinn, dieselben für das Charakteristische der Hysterie zu halten. Das Kennzeichen verlegte er in das Psychogene der Vorgänge. Da um die Zeit der Charcotschen Lehrtätigkeit für somatische Erscheinungen, welche durch Vorstellungen erzeugt werden, der Namen "Suggestion" aufkam, bedeutet es nur eine Umschreibung jenes Satzes, wenn man die Hysterie als gesteigerte Suggestibilität bezeichnet.

Der Begriff der Suggestion bedeutet etwas von außen an das Invididuum Herantretendes, eine Utberrumpelung des Verstandes. Daß man den Begriff der Autosuggestion aufbrachte, hat wahrscheinlich seinen Grund darin, daß man damit eine Lücke auszufüllen hoffte, welche die Erklärung durch die Suggestionen noch gelassen hatte. Ein Verlegenheitsbegriff, da er in seiner Zusammensetzung im Begriffsworte negiert, was das Bestimmungswort eben behauptet hatte, nach Hellpach ein barbaristisches Wort, das sich nach dem alten schlechten Gewobnheitsrecht der Pathologie auch heute noch überall einzustellen pflegt, wo Begriffe fehlen. Dieser Begriff der Autosuggestion wurde nur deswegen gebildet, weil man ja deutlich sah, daß die Suggestion weitaus nicht in allen 
Fällen als ätiologischer Faktor der Hysterie herangezogen werden konnte, andererseits man aber von der vorgefaßten Meinung von einer Zugrundelegung von Vorstellungen um so weniger lassen wollte, als es gelungen war, durch in der Hypnose beigebrachte Suggestion den hysterischen vollständig gleichende körperliche Erscheinungen zur Auslösung zu bringen. Auch das mag beigetragen haben, daß man aus der Art der Therapie ohne weiteres einen Rückschluß auf die Entstehung machte. Allerdings übersah man dabei, daß eine andere Einwirkung auf ein fremdes Individuum im gebräuchlichen Lebensverkehre als wirksame nicht in Betracht kommen kann als jene durch Vorstellungen, das Individuum aber, auf welches man einwirken will, die angestrebte Wirkung erst durch die psychologische Verknüpfung mit dem Affekte, welcher in jedem Falle sich den Vorstellungen verbindet, auf den Willen erhält.

Das Wesen der Suggestion liegt in dem Wegfalle der zum Zustandekommen einer Willenshandlung notwendigen natürlichen Motivation, also in dem Wegfall eines Inhaltes des Bewußtseins, durch welchen in diesem ein Akt zustande kommt, durch welchen es sich ein Ziel setzt. Es kommt also bei der Suggestion zu einer Zielsetzung, ohne daB jener verlangte Bewußtseinsakt vorausging, es erfolgt die Zielsetzung ohne Motivationsakt. Nur nebenher weise ich darauf hin, daß diese Klarlegung ebenfalls dazu führt, den inneren Widerspruch, den der Ausdruck Autosuggestion in sich birgt, aufzudecken. Auch das folgt daraus, da $B$, wie viele wollen, nicht jede thberredung gleichbedeutend sei mit Suggestion. Wenn man einen Menschen mit guten oder schlechten Gründen überzeugt und zu einer Handlung veranlaßt, dann hat eine solche Handlung mit Suggestion nichts gemein, weil sie aus toberzeugung auf Grund von normalerweise vorhandenen Motiven erfolgt, deren Verschiebung gegenüber dem objektiven Richtigkeitswerte das Wesen der Handlung als natürlich motivierte nicht tangiert. Bei ausgesprochenen Suggestionen kommt ein therzeugen überhaupt nicht in Frage, weil es sich um die Realisierung von Vorstellungen handelt, die aus irgendwelchen psychologisch verständlichen Gründen nicht die notwendige Kraftentfaltung zu einer Einflußnahme im Sinne des Überzeugers zu gewinnen vermögen.

Man wird unter allen Umständen die Suggestion in jeder Theorie der Hysterie zur Geltung kommen lassen müssen, weil die Tatsachen den Wert derselben nicht zu übersehen gestatten. Nur das wird in Abrede gestellt, daß der Weg der Suggestion der einzige sei, auf dem die hysterischen Symptome zustande kommen.

Unter den deutschen Autoren hat Möbi us den Begriff der Suggestion am deutlichsten als genetischen Faktor der Hysterie angesprochen: „Hysterisch sind alle diejenigen krankhaften Veränderungen des Kör- 
pers, die durch Vorstellungen verursacht werden." Die Wahrheit dieses Satzes ist unbestritten geblieben, aber die Auffasssung seines Autors, demselben sei ein exklusiver Sinn zu unterlegen, so daß krankhafte Veränderungen des Körpers, welche auf eine andere Weise zustande kommen, nicht als hysterische bezeichnet werden dürften, wurde von vielen Autoren zurückgewiesen. Denn in vielen Fällen vorkommender hysterischer Erscheinungen sind Vorstellungen sieher nicht vorhanden. Warum können Hysteriker, wenn man ihnen ihre Stigmata nachweist, ganz erstaunt und erschrocken sein? Die Einwendung, daß in solchen Fällen eben deutlich werde, daß die Vorstellungen vorhanden sind, aber der betreffenden Person nicht klar bewußt werden, widerspricht aller Psychologie des Vorstellungslebens. Denn es würde unerklärlich bleiben, daß eine so gewaltige Wirkung einer Vorstellung eintrete, ohne daß diese letztere bewußt, und zwar klar bewußt werde.

Mit der Verschiebung der Vorstellung in das Unbewußte, rückt man dieselbe überhaupt aus dem Felde der Suggestion hinaus, weil die Realisierung der hysterischen Produkte sich dann nicht mehr den diesen adäquaten Vorstellungen, sondern Vorstellungen ganz anderen Inhaltes anschließt. Eine Vorstellung im Unterbewußtsein könnte schon deshalb als Kausalitätsfaktor nicht geltend gemacht werden, weil dieselbe als eine Annahme gilt, welche zur Erklärung mangels einer Erkennungsmöglichkeit nichts beitragen kann. Die Lehre von den unbewußten und den unterbewußten Geistesprozessen ist ein bequemes Haus geworden, in welches man schließlich alles als glänzendes Meublement hineinstellen kann, was man dem kritischen Auge der Öffentlichkeit entrücken will.

Da die Hypnose hauptsächlich als jener psychische Vorgang gilt, in dem die Suggestion am meisten zur Geltung kommt, ist man auch weiter gegangen und hat die Hysterie als einen der Hypnose analogen Proze $\beta$ bezeichnet. Deswegen aber, weil ein Vorgang in der Hypnose durch Suggestion zustande kommt, muß doch noch lange nicht jedes Vorkommen desselben auf Suggestion beruhen. Weiter bedeutet es einen falschen FehIschluß, deswegen, weil die Suggestion in der Hypnose produktiv wird, jeden Prozeß, in welchem die Suggestion als realisierendes Moment in Betracht kommt, mit der Hypnose zu identifizieren, im gegebenen Falle die Hysterie als gleichbedeutend mit Hypnose zu bezeichnen oder gar als Art der Gattung Hypnose unterzuordnen.

Der Fehler im Schlußaufbaue liegt in jener Prämisse, welche das tatsächliche richtige Verhältnis zwischen Suggestion und Hypnose in dem Sinne, der besagt, in der Hypnose kämen die Wirkungen durch Suggestion zustande, durch ein vorgesetztes n ur in ihrem Sinne vollständig ändert. Nur in der Hypnose werden Suggestivvorstellungen 
realisiert. Diese Realisierung ist ein Charakteristicum der Hysterie. Folglich ist die Hysterie Hypnose.

Die Lehre von der Suggestion gelangte zu so allgemeiner Anerkennung, wie sie sie heute besitzt, großenteils deswegen, weil sie auf therapeutische Erfolge hinwies. Ich nahm schon die Gelegenheit wahr, auf das Fehlerhafte des Rückschlusses aufmerksam zu machen, der von einer Wirksamkeit in der Therapie auf die Genese gezogen wird. Ob die Suggestion auf dem Gebiete der Therapie wirklich so uneingeschränkt herrscht, wie es scheinen kann, ist eine um so schwerer zu entscheidende Frage, als die reine Wirkung der Vorstellung an sich kaum prüfbar ist. Denn nicht als solche wird sie vom Individuum im Bewußtseinsschatze auftretend gefunden, sondern stets schon mit der psychologisch von ihr nicht zu trennenden Begleitung des Affektes. Man hat gerade in der Therapie sich zu sehr daran gewöhnt, jede Art seelischer Beeinflussung Suggestion zu nennen. Dieser laxe Sprachgebrauch wirft den durch Utberzeugung erreichten Erfolg mit dem durch Uberredung erreichten zusammen, insbesondere dann, wenn neuro- und psychopathologische Eigenschaften, z. B. Neurasthenie, die Feststellung etwas erschweren, was auf Rechnung der einen und was auf Rechnung der anderen zu setzen sei.

Charcot hat nirgends eine Definition der Hysterie gegeben. Aber er benennt sie einmal die Psychose par excellence, ein andermal ein Leiden, welches zu drei Vierteilen als psychisches Leiden aufgefaßt werden müsse. Gegen die Auffassung im ersten Sinne sprechen alle Fälle von Hysterie, in denen dieselbe ohne geistige Störung als reine Neurose entgegentritt. Daß Charcot zu der Auffassung einer rein psychotischen Erklärung der Hysterie kam, liegt wohl an den Eigenheiten des Krankenmaterieals, an welchem er seine Studien machte und an der Anlehnung an Georget, der vor ihm am gleichen Orte und zum großen Teile am gleichen Materiale über das Thema der Hysterie gearbeitet hatte. Die zweite Stelle kann in der Kontroverse nicht verwertet werden, weil Charcot nirgends angibt, als was das vierte Viertel zu betrachten sei. Sogar für die traumatische Hysterie, für welche die deutschen Autoren dem Unfall eine materielle Wirkung auf das Nervensystem einräumten, hat Charcot nicht das Trauma, sondern lediglich die damit verbundene Gemütserschütterung als den Ausgangspunkt der anschließenden hysterischen Erkrankung angesehen, indem die mit dem Schreck verbundenen Vorstellungen als Suggestionen fortwirkten.

Den klarsten Aufbau der rein psychisehen Auffassung des Wesens der Hysterie hat die französische Theorie in den Darlegungen Ja nets gefunden, für den die pathologische Zerstreutheit der Hysteriker und die dadurch bedingte Einengung ihres Bewußtseinsfeldes die Grundlage des Krankheitszustandes bildet. Nach Ja net ist die Hysterie eine Geisteskrankheit, welche zu der Gruppe der Krankheiten durch cerebrale Erschöpfung gehört, weshalb sie auch durch psychische Symptome charakterisiert sei, während die physischen Krankheitserscheinungen unbestimmt bleiben. „Das Hauptsächlichste ist eine Abschwächung der Fähigkeit zur psychologischen Synthese, eine Willensschwäche, eine Einengung des Bewußtseins, welche sich auf eine eigenartige Art kundgibt $\left.{ }^{1}\right) .{ }^{\text {": }}$

1) Janet, Der Geisteszustand der Hysterischen. (Übersetzt von Kahane). 
Dieselbe komme zustande, indem eine gewisse Zahl elementarer Empfindungsvorstellungen nicht mehr wahrgenommen wird, wodurch dieselbe aus der persönlichen Auffassung ausgeschaltet erscheine. Dieser Vorgang respektive Defekt erzeuge eine Tendenz zur dauernden und völligen Spaltung der Persönlichkeit; die zu den verschiedenen Persönlichkeiten gehörenden psychologischen Tatsachen lösen sich gegenseitig ab oder bestehen nebeneinander. Darauf beruht auch, daß außerhalb der Kontrolle des Selbstbewußtseins ,bestimmte parasitäre Vorstellungen" sich entwickeln, welche sich durch verschiedenartigste, nur scheinbar ausschließlich psychische Störungen kundgeben. Der Effekt jener Schwäche der psychologischen Synthese sei eine Art von geistiger Zersplitterung.

Janet nimmt also zwei Grundlagen der Hysterie an: die Abspaltung von Bewußtseinsanteilen und die Schwäche des Kombinationsvermögens (de la synthèse psychologique). Indem Möbi us sich bemühte, zwischen diesen beiden Faktoren eine Verbindung ausfindig zu machen, kam er dazu, die BewuBtseinsspaltung als das Primäre der Kombinationsschwäche als dem Sekundären gegenüberzustellen. In der Analyse der Psychologie jener Vorgänge gelangte Ja net über den Vorstellungsbegriff nicht hinaus, weil er in der Empfindung nichts Psychisches sah, sondern nur den physischen Begleitvorgang der Empfindungsvorstellung. Hätte sich Janet weiter gefragt nach dem Grunde jener Bewußtseinsspaltung und Kombinationsschwäche, dann hätte ihn die psychologische Analyse zur Erkenntnis geführt, daß beide ihre gemeinsame Fundierung in der Wirkung von Gemütsbewegungen haben, die erfahrungsgemä $B$ die Beziehungen zwischen Außenwelt und Bewußtsein zu lenken vermögen.

Für die Auslese bestimmter körperlicher Erscheinungen glaubt Janet mit unbewußter Eklektik der Kranken auskommen zu können, welche mit dem Alter in gesetzmäßigem Zusammenhange stehen soll: eine Annahme, welche nach den Feststellungen vieler Autoren mit den Tatsachen in Widerspruch steht.

Während Janet gerade im Fehlen bestimmter psychologischer Vorstellungselemente die Ursache der Krankheit sucht, haben andere fast umgekehrt gerade im Herausheben bestimmter Vorstellungskomplexe gegenüber den anderen ihre Begründung finden wollen. Während dort die Schwäche der Empfindungsvorstellungen und schon gar deren Fehlen einen Willensakt überhaupt nicht in Erscheinung treten läßt, soll hier eine pathologische Störrigkeit gegenüber aller Vernunftmotivation vorhanden sein.

Diese beiden Ansichten haben das Gemeinsame, daß sie die rein psychologische Theorie der Hysterie an den Ausgangs- und an den Endpunkt ihrer Erörterungen setzen. Insofern sie allein die ihnen passenden, tatsächlich durch Vorstellungen begründeten hysterischen Krankheitserscheinungen in Betracht ziehen, hat einer, der eine andere Theorie festhält, keinen Grund, sich in eine Kontroverse zwischen beiden Meinungen einzumischen, zumal er doch die Prämisse überhaupt nicht gelten läßt, daß hysterische Krankheitserscheinungen nur durch Vorstellungen hervorgerufen werden. In diesem Punkte gilt die Abweisung für beide Theorien in der gleichen Weise. Ob man annimmt, wegen fehlender Vorstellungen komme es zum Fehlen eines zu ihrer Realisierung notwendigen Willensaktes, oder die andere Meinung vertritt, ein einseitiges Festhalten an dieser oder jener Vorstellung oder Vorstellungsreihe ändert nach der angekämpften Richtung hin nichts, so daß für beide 
Theorien gemeinsam die Zurückweisung gegeben ist, wenn die beiden gemeinsame Voraussetzung widerlegt ist.

Janet selbst scheint mir die zweite Ansicht abzuweisen, wenn er davor warnt, fixe Ideen zur Grundlage aller hysterischen Erscheinungen zu machen. Indem er nämlich seine Theorie von der Einengung des Ich-Bewußtseins zur Erklärung der Anästhesien heranzieht, betont er, daB die cerebrale Tätigkeit in ihrer Gesamtmenge verringert zu sein scheine. Es könne noch jedes einzelne corticale Zentrum in funktionelle Erregung versetzt werden, aber die verfügbare nervöse Kraft könne nicht mehr mehrere Zentren zu gleicher Zeit wecken. Wahrscheinlich seien diese corticalen Störungen in die Assoziationszentren zu verlegen, während die Schwäche aller anderen corticalen Zentren als sekundäre Schwäche aufzufassen sei. Die Empfindungen und Vorstellungen des kranken Individuums besitzen den gröBten Einfluß auf die Verteilung des verfügbaren Restes der Aktivität, woraus sich der Einfluß der fixen Ideen erkläre. Da diese Erscheinung keine bei Hysterie allgemeine sei, könne sie nicht zur Erklärung des Ursprunges der Krankheit herangezogen werden.

Unter jenen Autoren, welche sich in der energischsten und lebhaftesten Art für die Suggestionsgenese der Hysterie eingesetzt haben, nimmt einen Platz in den Vorderreihen $\mathrm{Babi} \mathrm{ski}^{1}$ ) ein. Ein großer Teil seiner wissenschaftlichen Arbeit galt dem Thema der Zergliederung der Hysterie. Er gelangte zúm Schlusse, daß als hysterisch nur das bezeichnet werden könne, was durch Suggestion erzeugt und durch Persuasion wieder entfernt werden könne. Den Begriff der Persuasion setzt er gleich dem der Suggestion vernünftiger Dinge. Er weist jeden Einfluß von Gemütsbewegungen auf die Genese der Hysterie ab und bemerkt, da $B$ in keinem der Fälle, in deren Ätiologie diese eine Rolle spielen, es sich um Hysterie handle.

Diese Trennung von suggestiver und gemütlicher Beeinflussung ist psychologisch undurchführbar, da ja die Suggestion ebenso wie die Persuasion immer aus das innigste mit Gemütsbewegungen verknüpft ist und nach aller psychologischen Erfahrung erst die letzteren der Vorstellung jene Triebkraft verleihen, die ihr eine Einwirkung (in positivem oder negativem Sinne) auf das Willensvermögen ermöglichen. Unter den vielen aus der Front unserer Anstalt zugewachsenen Kranken befand sich eine beträchtliche Zahl von solchen mit ersten hysterischen Zufälligkeiten. Eine suggestive Einflußnahme konnte in vielen gar nicht statthaben, weil eine verbale Verständigung weder direkt noch indirekt (durch einen Dolmetsch) möglich war, ich es andrerseits vermied, durch meine Gesten eine suggestive Wirkung auszuüben. Gleichwohl war es durch Anwendung des faradischen Stromes ohne weiteres möglich (wenn auch nicht immer), die hysterischen Symptome in kurzer Zeit zum Schwinden zu bringen. Offensichtlich war es bei dieser Wirkung wenigstens nicht eine Vorstellung allein, welche den Effekt der Lösung zur Folge hatte und schon gar nicht jene, welche dem Effekte adäquat war, sondern die schmerzgefühlsbetonte Empfindung.

Diese psychologischen (oder richtiger psychopathologischen) Theorien gehen über den Begriff der Vorstellung nicht hinaus, stellen ihn als eine

1) Babinski, Démembrement de l'hystérie traditionelle. 
elementare Einheit hin, wenn nicht explicite, so doch implicite, da sie keinen Versuch machen, denselben zu zerlegen. Ein solches Vorgehen führt zu einer Lösung, die nur im Sinne der Assoziationspsychologie als eine abschließende betrachtet werden kann.

Gegenüber dieser assoziationspsychologischen Auffassung hat Möbi us hervorgehoben, daß es sich nicht um reine Vorstellungen, sondern um Vorstellungen plus Wollen handelt, um ein psychisches Gebilde, welches er seelisches Radikal nennt. Der Inhalt dieses Radikales soll beim Hysterischen ein Nichtkönnen sein. Es muß also, gemessen nach den allgemeinen psychologischen Begriffen, dasselbe als eine Änderung der ps ychophysischen Funktionalität sich darstellen, nicht wie die rein psychischen Theorien notgedrungen annehmen, ein Nicht-Wollen-Können.

Den psychischen Theorien gegenüber ist darauf hingewiesen worden, daß zahlreiche hysterische Krampf- und Lähmungserscheinungen sowie hysterische paroxystische Zufälligkeiten Folgewirkungen von emotivem Shock sind, welche nur durch intense Empfindungen, also rein sensorielle Gefühle verursacht sind. In allen diesen Fällen ist die in einer körperlichen Fixierung sich ausdrückende hysterische Konversion vollständig unabhängig von einer affektbetonten Vorstellung; in ihnen wurde überhaupt keine Vorstellung und keine assoziative Vorstellungsreihe geweckt. Beidenselben kann auch eine Erinnerungsvorstellung zur Erklärung nicht herangezogen werden. Diese Fälle sind ganz besonders geeignet, nachzuweisen, daß dem Ausdrucke ,durch psychische Vorgänge" in der in den Eingang gestellten Definition der Hysterie eine Ausdehnung gegeben werden müsse, die über den nächstliegenden Sinn der Worte hinausgeht, wenn man es nicht vorzieht, mit Rücksicht auf die Tatsachen der psychologischen Analyse diese heute noch übliche Definition fallen zu lassen.

Diese Erscheinung, daß nur aus Empfindungen entstehende, also sensorielle Gefühle, die Auslösung werden für hysterische Erscheinungen, ist in der gegenwärtigen Kriegszeit klar vor die Augen gestellt worden. In den abgelaufenen zwei Jahren habe ich eine große Zahl von hysterischen Krankheitsfällen gesehen, bei denen dieselben nur durch die schweren Kriegstraumata, insbesondere durch Minen- und Granatenexplosionen in Erscheinung gebracht wurden. Zum größten Teile bieten gerade diese Fälle die hysterischen Stigmata in solcher Klarheit, daß man unter den anderen, durch Wahrnehmungs- und Erinnerungsvorstellungen ausgelösten Fällen kaum annähernd eine gleiche Präzision im Krankheitsausdrucke findet. In der Friedenszeit konnte ich analoge Fälle unter den Frauen beobachten, während unter den Männern kaum ähnliche zu finden waren. Wollte man hier mit einer psychischen Erklärungstheorie das Auskommen zu finden hoffen, dann stieße man wohl immer und immer wieder auf unüberwindliche Schwierigkeiten in der psychologischen Analyse. Diese Erkrankungen führen zur Festigung 
der Auffassung, daß das Zustandekommen der hysterischen Symptome durch Beeinflussungen sich vollzieht, die aus den unwillkürlichen körperlichen Ausstrahlungen von Gemütsbewegungen entspringen.

Diese Fälle beweisen, daß zahlreiche hysterische Krampf- und Lähmungserscheinungen sowie hysterische Paroxysmen Folgewirkungen eines emotiven Shocks sind. Die bei den meisten unserer Kriegspatienten vollständig unzureichende Anamnese gestattete mir nicht die Bildung eines festen Urteiles, ob bei den meisten oder allen oder nur wenigen der Betroffenen für die Vorzeit eine hysterische Veranlagung nachgewiesen werden kann. Da ich deswegen keinen Anlaß habe, von der allgemeinen Annahme abzugehen, meine ich, daß jene Erscheinungen hauptsächlich dann eintreten werden, wenn auf dem Boden hysteriseher Stimmungsanomalien ein verhältnismäßig geringfügiger Zuwachs emotioneller Erregung Entladungen der Gehirnzentren herbeiführt. Hier ist die Auslösung der hysterischen Erscheinungen von Vorstellungen ganz unabhängig, weil gerade die Hemmung der Assoziationsvorgänge die Folgewirkung der Emotion war. Ebensowenig wie um aktive Vorstellungen kann es sich hier um Wirksamkeit bestimmter Erinnerungsbilder handeln.

Die zweifache Möglichkeit der Hervorrufung hysterischer körperlicher Erscheinungen durch Vorstellungen und durch sensorielle Vorgänge führt dahin, den Grund der Entstehung jener Erscheinungen in einem Faktor zu suchen, welcher beiden genannten psychischen Vorgängen gemeinsam ist, der bei beiden vorkommt. Dieser gemeinsame Faktor ist der Affekt, dessen Genese eine doppelte psychologische Ätiologie haben kann: aus den Vorstellungen oder aus den Empfindungen. Die zweite Art der Ätiologie läßt nämlich eine Erklärung, wie man sie für die erste zurechtgelegt hatte, überhaupt nicht zu, wodurch die Notwendigkeit entsteht, entweder die Gleichheit der verursachten Erscheinungen zu leugnen oder einen weder mit den Vorstellungen noch mit den Empfindungen identischen, denselben übergeordneten Faktor oder einen mit denselben in gleicher Weise verknüpften psychischen oder psychologischen Faktor als verursachendes Moment einzusetzen. Die Leugnung der Gleichheit widerspräche aller Erfahrung, der gesuchte übergeordnete Faktor existiert nicht. Der dritte Weg führt zu dem eben angeführten Resultat.

$\mathrm{Zu}$ den psychischen Theorien gehören selbstverständlich auch jene, welche das Wesen der Hysterie in einer Eigenart des Affektlebens erschöpft sehen. Eine eigene Widerlegung derselben ist nicht notwendig, wenn man das schon Gesagte über die Unmöglichkeit des Psychischen überhaupt, die normalen Ausdrucksbewegungen in abnormale umzusetzen, ohne daß das Psychische in sich eine Abänderung seiner Seinsform erfahren hat, nicht aus dem Auge verliert. Diejenigen, welche, 
um jene Eigenheit des Affektlebens zu erklären, auf die den Affekten zugrunde liegenden Vorstellungen hinübergreifen, geraten damit in dieselben Schwierigkeiten, wie ich sie für die Suggestionstheorie der Hysterie auseinandersetzte. Andere, welche auch das niedere, an die Organempfindungen gebundene Affektleben einbeziehen, öffnen sich damit noch nicht die Pforte zur Erkenntnis, wie gerade das Atypische der Ausdruckserscheinungen zu erklären ist, solange sie den physischen Riegel vor der Psychologie der Hysterie nicht zurückgeschoben haben. Im eigenen Vaterlande erstand den Hauptverteidigern der psychischen Theorie ein scharfer Gegner.

Bernheim hat aus der Genese der Hysterie das psychische Element als notwendigen kausalen Faktor ausgeschieden. Nach ihm stellen sich die hysterischen Erscheinungen, welche als körperliche Krankheitserscheinungen zur Erscheinung kommen, als reine Reflexe dar. Personen, welche sich in einem besonders gesteigerten reflektorischen Erregungszustande befinden, nennt er hystérisables, gleichgültig, ob sie geistig gesund oder krank sind. Diese Neigung zu einer gesteigerten Reaktion sei erst dann als hystcrisch anzusehen, wenn sie einen besonders hohen Grad erreicht hat und eine besonders leichte Ansprechbarkeit der Reflexe gegeben ist. Die hysterischen Stigmata schaltet er aus dem Bilde dieser Reaktionsform aus und weist ihnen eine große Selbständigkeit zu, indem er sie als Psychoneurosen der Sensibilität und Motilität auffaßt, die durch Suggestion und Autosuggestion leicht hervorgerufen werden können. Da sie auch bei anderen als hysterischen Individuen sich finden, hängen sie nicht von der Hysterie, sondern von den zu ihr hinzutretenden psychischen Erkrankungen ab. Das Wort Hysterie müsse den Krisen vorbehalten werden, die als gesteigerte psychophysiologische Reaktionen aufzufassen seien.

Breuer stellt das psychische Moment des Affektes nur für den Anfang der Krankheit als notwendig hin, während es später verschwinden soll, indem dann die ursprünglich affektive Vorstellung nicht mehr den Affekt, sondern nur den abnormen Reflex hervorrufe. Diese Meinung läßt also gerade jenen Vorgang verschwinden, welcher nach aller Psychologie nicht fehlen darf, damit es zum Auftreten einer Handlung komme, die als von einer Vorstellung ausgelöst anzusehen ist, Fine solche Loslösung einer Vorstellung vom Affekte ist eine Unmöglichkeit. Jede auftretende Vorstellung wird sofort von dem adäquaten Affekte umgeben. Denken und Fühlen sind zwei untrennbar verbundene Seelenmerkmale; eines ohne das andere kann nicht existieren. Es ist darum undenkbar, daß eine Vorstellung, nach noch so langer Gewöhnung oder nach noch so häufiger Wiederholung, jemals ohne ihre zugehörige Gefühlsbetonung auftrete. Eine Abschwächung des Affektes kann eintreten und wird nach psychologischer Erfahrung im Laufe der Zeit eintreten, eine Verschwinden des Affektes nie. Die Ansicht Bre uers begegnet also einer unüberwindlichen psychologischen Schwierigkeit.

Eine ganz besondere Stellung unter den Hysterietheorien nimmt jene ein, welche die Hysterie zu einer reinen Willenspsychose macht. Sokolowski1) hat als Anhänger derselben die Hysterie bezeichnet als: Kranksein als Äquivalent des psychischen Gleichgewichtes bei subjektiv empfundener Unzulänglichkeit entarteter Individuen. In dieser Definition wird vor allem übersehen, daß es sich bei der Hysterie nicht um

1) Sokolowki, Zentralbl. f. Nervenheilk. 1896. 
ein Kranksein einfachhin, sondern um eine ganz bestimmte Form des Krankseins handelt, welche durch das Auftreten von körperlichen Erscheinungen bei Affekten charakterisiert ist, von Erscheinungen, welche nicht als der typische Ausdruck der letzteren anzusehen sind. Der Wille kann nur jene körperlichen Ausdruckserscheinungen hervorbringen, welche bereits in dem Körper ihre Grundlegung haben, so daß der Wille in der Hervorbringung an das Physische gebunden scheint, also auch nur dann eine abnorme, vom Typus abweichende körperliche Ausdruckserscheinung auftreten lassen kann, wenn dieselbe in den Eigenheiten des Nervensystems begründet ist. Für gewöhnlich genügt ja der Wille zum Kranksein nicht, um krank zu sein, auch dann nicht, wenn das psychische Gleichgewicht schwer gestört ist, auch dann nicht, wenn die Unzulänglichkeit recht intensiv empfunden wird und schließlich auch bei einer Entartung im allgemeinen nicht. Es wird eine ganz bestimmte Entartung gefordert, und zwar eine nervöse, nicht eine rein psychische Entartung. Diese Theorie hat das Bestreben, die Hysterie im Begriffe der psychischen Degeneration aufgehen zu lassen. Die Intention schlechtweg krank zu sein, bildet das Primäre, während die Krankheit, ihr Charakter und ihre Symptome erst als Ausflüsse dieser Intention sich ergeben oder zwar als schon vorhanden in ihren Dienst treten und pathognomonisch erst in zweiter Linie stehen. Nach meiner Meinung besteht zwischen den beiden Disjunktionen dieses Satzes ein wesentlicher Unterschied. Im ersten Falle erscheint das ganze Krankheitsbild als Resultat des Wollens, im zweiten aber erscheint die Selbständigkeit des körperlichen Krankheitsbildes ganz ebenso sichergestellt, wie dort, wo dasselbe ausdrücklich als der Ausdruck eines eigenen neurotischen Prozesses aufgefaßt wird. Diese erstere Auffassung würde sich mit dem Janetschen Fehlen der psychologischen Synthese decken, zumal (nach Sokolowski) der Wille einen assoziativen Prozeß bedeuten soll, dessen Korrektheit lediglich von der Klarheit und Korrektheit der vorhandenen Erinnerungsbilder und von der Exaktheit, mit welcher sie zitiert werden, abhängig ist, darin aber würde sie über Ja net hinausgehen, daß sie alles Neurotische aus dem Begriffe der Hysterie ausscheidet.

Als die erkennbaren Bestandteile jeder menschlichen Handlung bilden Affektursache und Affektausdruck die beiden Enden einer Reihe, welche ihre psychologische Verbindung durch den aus der Innenerfahrung bekannten Affekt findet, der im Falle der Hysterie mit der Affektursache kausal, mit dem körperlichen Phänomen funktional verbunden ist. Was tatsächlich vorgefunden wird, ist die Inadäquatheit zwischen Affekt. ursache und Affektausdruck. Ob nun ein Mißverhältnis zwischen Affektursache und Affekt oder zwischen Affekt und Affektausdruck besteht, hat die psychologische Untersuchung zu entscheiden. 
Im ersten Falle ergibt sich die von einer Reihe von Autoren als Dissoziation, im zweiten die von anderen als psychophysische Disproportionalität bezeichnete Erscheinung. Auf die erstere Deutung führen alle Theorien hin, welche im Psychischen das Wesen der Hysterie erschöpft haben wollen, während zur zweiten alle jene hinneigen, welche für die hysterischen Körpererscheinungen eine rein psychische Genese als nicht zureichend zur Erklärung halten und meinen, daß das Abweichen derselben von der funktionellen Norm eine eigene Erklärung nur aus den Innervationsverhältnissen, also aus einem physischen Momente, finden könne. Die nervenmechanische Erklärung Binswangers trägt diesem Postulate voll und ganz Rechnung. Hellpach rügt an derselben, daß sie sich zu weit in den Bereich der nervenmechanischen Theorie hinein entferne, weil "Innervationsvorgänge hypothetische Prozesse sind, von denen noch niemals jemand etwas beobachtet hat". Die Definition Krae pelins sagt ihm mehr zu. Den Ausdruck : psychophysische Disproportionalität bei Binswanger und Hellpach hat Skliar dahin gedeutet, es solle mit ihm eine Inkongruenz zwischen Reizstärke und psychischer Reaktion bezeichnet werden, weil mit der Zunahme des Reizes beim Hysteriker in vielen Fällen gar keine oder zuweilen gar eine entgegengesetzte Reaktion erfolge. Das Wort selbst fand ich bei Binswanger nicht. Er redet von,,Störungen des Gleichgewichtes zwischen den erregenden und den hemmenden Vorgängen innerhalb der Zentralnervensubstanz", ,,von Störungen im Ablaufe der Vorgänge der materiellen Reihe einerseits und jener der geistigen Reihe andererseits". Solche Redeweisen können wohl noch für fehlende Reaktion, aber wohl nur durch gewaltsame Interpretation für eine der normalen entgegengesetzte Reaktionsart angewendet werden. Hellpach bezeichnet als psychophysische Disproportionalität die Störung der normalen Zuordnung der Ausdruckserscheinungen zu den Gefühlserlebnissen, welche nach drei Richtungen: als qualitative, intensive oder extensive sich äußern könne. Die einzelne Störung könne nur nach einer, nach zwei oder nach allen drei Richtungen erfolgen. Nur die qualitative Störung könnte an sich als eine der Norm entgegengesetzte Reaktion betrachtet werden. Hellpach erklärt dieselbe aber ausdrücklich nur als eine unter den normalerweise auftretenden Reaktionen nicht vorkommende und hebt hervor, daß unter außergewöhnlichen Umständen und bei schweren Affekten die Vorbilder aller jener in der Hysterie vorkommenden Erscheinungen gegeben sind.

Besteht ein wesentlicher Unterschied zwischen der Auffassung Binswangers und jener $\mathrm{Krae}$ pelins, nach welcher als das wesentliche Kennzeichen der Hysterie zu betrachten ist die ,außerordentliche Leichtigkeit und Schnelligkeit, mit welcher sich psychische Zustände in mannigfaltigen körperlichen Störungen wirksam zeigen?" Diese wie 
jene enthalten sich jeden Urteiles über die Art des in Betracht kommenden Nervenprozesses. Binswanger hat allerdings seiner psychologischen Grundanschauung, dem psychophysischen Parallelismus, auch bei dieser Gelegenheit der Feststellung des Charakters der hysterischen Veränderung Ausdruck gegeben, indem er das Wesen der hysterischen Veränderung darein verlegt, daß die gesetzmäßigen Wechselbeziehungen zwischen der psychischen und materiellen Reihe gestört sind, indem einerseits für bestimmte Reihen materieller Rindenerregungen die psychischen Parallelprozesse ausfallen oder nur unvollständig durch jene gedeckt werden, andererseits einer materiellen Rindenerregung ein Ubermaß psychischer Leistung entspricht, das die verschiedensten Rückwirkungen auf die gesamten Innervationsvorgänge, die in der Rinde entstehen oder von ihr beherrscht werden, hervorruft. Hellpach würde seinen Tadel wahrscheinlich nicht ausgesprochen haben, wenn er jene Stelle in der Monographie Binswangers (S. 15) beachtet hätte, an der Binswanger auch im Ausdrucke Krae pelin so nahekommt: , Die pathologische Mehrleistung an psychischer Arbeit gibt sich demnach nicht allein in einer Steigerung der Gefühlserregungen, in einer Verschärfung der Empfindungen oder in dem Auftauchen überwertiger Vorstellungen kund, sondern auch d urch die a uffallende Leichtigkeit, mit welcher die psychischen Vorgänge, vor allem die Vorstellungen, auf alle hysterischen Funktionsstörungen einwirken."

Die psychophysische Disproportionalität findet nach Hellpach darin ihren Ausdruck, daß die normale Zuordnung der Ausdruckserscheinungen zu den Gefühlserlebnissen qualitativ, intensiv und extensiv - jeweils in einer oder in zwei oder in allen diesen Richtungen - gestört erscheint. Ich deutete schon cinmal an, daß die für Hysteric charakteristische Störung cire qualitative ist, während die intensive und extensive Störung die Hysterie mit anderen Neurosen und Psychosen gemeinsam hat. Auf welche Grundeigenschaft der nervösen Bahnen dieselbe zurückzuführen sei, kann nach meiner Ansicht nicht gesagt werden. Das hindert aber nicht, daß die Forderung einer nervösen Störung in ihrem Rechte bestehen bleibe.

Der Versuch, jenen schon öfter crwähnten Innervationszustand in sciner Qualität zu bestimmen, hat bei Sollier die Ansicht ausgebildet, derselbe bestche in einem Zustand des Schlafes einer mehr oder minder großen Zahl von Hirnzentren, welche einzeln oder mit anderen, gleichzeitig oder nacheinander davon befallen werden können. Die Ursache dazu liege am häufigsten in einer Erregung, welche das Individuum gegen das Entstehen und die Entwicklung von Bewußtsein gefühllos mache. Als die Hauptursache der Hysterie bczeichnet er cine psychische Störung, einen Zustand von Erstarrung und mchr cder wenigcr tiefcm Schlafe der crwähnten Hirnzentren, welcher durch die Erregung auggelöst und in Szene gebracht wird. Diese Ansicht weicht schon in einem bemerkenswerten Punkte der Ausdrucksweise von der seiner engeren französischen Kollegen ab, da sie nie von jenen Zentren spricht, wclche schon damals als die Vorstellungezentren bekannt waren, nämlich von den Hirnrinden zentren. Sollier hat sclbst die Abweichung scincr Ansicht von jener Janets erkannt und klargestellt: Ja net sehe bei der Hyst rie nur eine psychologische Störung, er aber eine physiologicshe; von jenem sei die elementare Sensibilität in der Hysterie als erhalten angenommen, ihm gelte das 
Fehlen der Gehirnsensibilität als wesentlich. Darum sei dieselbe bei Janet eine Folge der persönlichen Synthese, während bei ihm von vornherein und primär die Gchirnsensibilität an sich ins Auge zu fassen sci. Sollier tritt der Meinung entgegen, als sei die Vorstellung (l'idée) imstande, einc hycterische Störung hervorzurufen. Erst die durch sie bestimmte Erregung bringe das zuwege. Ob nun jene Emotion durch eine von außen kommende Vorstellung (par une image extérieure) oder durch eine Erinnerungsvorstellung (par une image ancienne, une idée, un souvenir) verursacht werde, sei von keincr Bedeutung. Der dadurch bewirkte Unterschied sei nur cin Unterschied in der Stärke der Erregung.

$\mathrm{Zu}$ dieser Theorie Solliers ist vor allem anzumerken, daß er mit der Heranziehung des Schlafzustandes zur Erklärung nichts beigetragen hat, da uns über das Wesen des Schlafes bis heute nichts Sicheres bekannt ist. Die einen sehen den Grund in einer Anämie des Gehirnes, andere in einer Hyperämie. Es ist heute wissenschaftliches Gemeingut, daß der Schlaf zwar ein Aufhören des Selbstbewußtseins und eine Unterbrechung des zeitlichen Zusammenhanges der psychischen Erlebnisse, aber kein Schwinden des Bewußtseins schlechthin bedeutet, da ja im Traume sich psychisches Geschehen abwickelt. Sollier hat an der Theorie Janets, deren Genialität er anerkennt, ausgesetzt, sie sei nur eine Feststellung. Und die seine? Was Sollier zum einfachen Tatbestande, daß bei den Hysterischen zuzeiten bestimmte psychische Komplexe nicht verfügbar zu sein scheinen, hinzufügt, ist Hypothese und nicht einmal gut begründete. Denn fürs erste : können die hysterischen Körperphänomene aus der Funktionsunfähigkeit bestimmter Hirnpartien erklärt werden? Z. B. die Tastempfindungen? Fürs zweite jene schon gekennzeichnete Schlafhypothese? Mir weckte die Theorie Solliers den Eindruck, als habe dieser Autor den Begriff der Hysterie einfach durch einen anderen, ebensowenig klaren und erklärten ersetzt: den des partiellen Somnambulismus. Was ist partieller Somnambulismus?

Den Mangel der Sollierschen Theorie, eine Erklärung der Hysterie mit einem noch unerklärten Begriffe versucht zu haben, suchte Oppenheim zu vermeiden, indem er eine Mechanik des nervösen Geschehens angab, welche fähig und genügend sein soll, die Grundlegung der hysterischen Symptomatologie zu bilden. Die Grundlage der Hysterie sei in einer verminderten inneren Reibung der Moleküle des Nervensystems zu suchen. Oppenheim selbst hat seine Ansicht als einen physiologischen Deutungsversuch bezeichnet. Als mehr kann sie auch nicht angesehen werden, da bei dem heutigen Stande der Gehirnphysiologie jede physiologische Deutung notwendig in radikaler Theorie sich erschöpfen muß. Diese Theorie hat mit jenen von Ona noff und Pitres gemeinsam, da $\beta$ sie als das Wesentliche an der Hysterie einen krankhaften Gehirnzustand ansieht, aus dem sich zahlreiche pathognostische Störungen der Empfindung und Bewegung auch ohne psychogene Einmischung ergeben.

Geboren wurden diese Auffassungen aus der seinerzeit noch mehr als heute vertretenen Anschauung, daß eine Theorie, welche auf objektiven Wert Anspruch erheben will, nur anatomischen Charakter haben könne. 
Diese Anschauung wurde in weitreichendem Maße von Meynert und Flechsig auf die Nerven- und die Geisteskrankheiten übertragen, nach denen alles Psychische sich in einer mosaikartig gemusterten Hirnrinde erschöpfte. „,Die schlechte Gewohnheit ist so eingewurzelt, daß Männer, die berufen wären, psychologische Fragen vom Fleck zu bringen, offenbar an nichts Psychisches ohne die gleichzeitige Halluzination eines Stückes Gehirn zu denken vermögen. Neun Zehnteln aller psychologischen Einfälle und Interpretationen wird der Weg mit der Übersetzung ins Anatomische oder, was neuerdings mehr in Mode kommt, ins Physiologische verschüttet." (Hellpach.)

Die Psychopathologie scheint in ganz besonderer Weise zur Beleuchtung der Hysteriefrage beitragen zu können. Nur darf man nicht von vornherein jeden Fall geistiger Erkrankung, bei dem hysterische' Symptome sich geltend machen, als hysterische Psychose bezeichnen und damit einen Standpunkt einnehmen, welcher, trotzdem er weder logische noch psychologische Berechtigung hat, von einem großen Teile der Psychopathologen vertreten wird. Auch der Vorschlag Krafft-Ebings und Schüles, als hysterische Psychose eine bestimmte Geistesstörung abzugrenzen, nämlich jene, bei der ausgesprochen hysterische Symptome vorhanden sind, geht dem gemachten Vorwurfe der Nichtberechtigung nicht aus dem Wege. Daher finde ich die Bezeichnung hysterische Geistesstörungen für Psychosen bei Individuen, welche Hysteriker sind, ohne weiteres Vordringen in die Eigenart der Psychose unzukömmlich. Der Begriff des hysterischen Irreseins unterliegt bei solcher Auffassung naturgemä $\beta$ weiten Schwankungen und gerade von psychiatrischer Seite ist man gegen ihn aufgetreten.

Man stelle sich vor, wie viele von den Psychosefällen, welche nach klinischer Betrachtungsweise einer anderen bestimmten Form von Geisteskrankheiten zugewiesen werden, nach der vorerwähnten Auffassung aus derselben ausgeschieden werden müßten, wie sehr aber gleichzeitig durch sie das Bild der Hysterie verschwommen gemacht und verzerrt würde.

Die Stellung in der Frage Hysterie-Geisteskrankheiten ist aussehlaggebend fundiert in der grundsätzlichen Lösung: Ist Hysterie Psychose oder Neurose? Ist Hysterie eine psychische oder psychophysische Krankheit? In ersterem Falle, in welchem die psychische Disposition allein das Fundament der Krankheit bildet, bleibt nur die Wahl, anzunehmen, daß zwei in ihren Merkmalen verschiedene Geisteskrankheiten gleichzeitig die Psyche beherrschen oder bei Vorhandensein von hysterischen Stigmatis die anderen Merkmale in ein psychologisches Prokrustesbett zu spannen und auf diese Weise künstlich eine Konformität dieser mit jenen zu erzwingen. Die erste Annahme ist nicht durchführbar, 
weil sie der psychopathologischen Erfahrung widerspricht. Die zweite wird dem Widerspruche nicht entgehen, zum Zwecke der Erreichung eines vorgefaßten Zieles den Erfahrungen Gewalt angetan und eine Prämisse des Schlusses zielentsprechend zugestutzt eingesetzt zu haben.

Diesen Schwierigkeiten weicht die zweite, das Wesen der Hysterie betreffende Annahme ungezwungen aus. Sie spricht von Verbindung einer Neurose mit einer Psychose und hat es deswegen auch gar nicht nötig, die Einschätzung der nicht ihrem Charakter vollständig entsprechenden psychischen Symptome erst zu modellieren.

Man muß, sagt Charcot, die Hysterie für das nehmen, was sie ist, nämlich für eine Geisteskrankheit par excellence. Tut man das, so verstehe man auch, meint Gilles de la Tourette, daß es notwendigerweise einen besonderen hysterischen Geisteszustand geben muß, der, wie die dauernden Stigmata, zum allgemeinen Krankheitsbilde gehört.

Charcot selbst hat den für die Hysterie anzuwendenden Begriff der Geisteskrankheit übrigens gar nicht in dem gebräuchlichem Sinne einer zeitlich begrenzten Erscheinung verstanden, sondern in dem, welcher eine psychopathologische $\mathrm{Ab}$ weichung von der Norm einfachhin bedeutet, eine psychopathologische Charakteränderung. Neben dieser kann eine Geisteskrankheit im engeren Sinne ganz gut selbständig auftreten und bestehen, was bei der anderen Auffassung nicht möglich wäre, da die Einfachheit der Psyche unüberwindliche Schwierigkeiten entgegenstellte. Die Hysterie wäre dann bei allen nicht für sie spezifischen Geisteskrankheiten nur etwas denselben einen bestimmten Ton und eine ganz eigentümliche Färbung Gebendes, was bei jeder derselben in derselben Weise auftreten und darum aus allen heraus als von jeder cinzelnen Verschiedenes und Selbständiges erkannt werden kann.

Wenn die Krankheitsgeschichten, welche er an seinen Demonstrationsfällen erläutert, in diesem Sinne aufgefaßt werden, dürften sich damit jene Vorwürfe erledigen, welche man Charcot und seiner Schule machte, weil sie hysterische Erscheinungen an verschiedenartigen geistigen Erkrankungen und nicht an gleichartigen demonstrierten. Vielleicht lag es in der Absicht Charcots, seine Meinung von der Hysterie als psychopathologischer Charakterveränderung auf diese Weise zu bekunden, indirekt zu tun, was er direkt nicht aussprach. Die Hysterie bliebe dann nicht nur in der theoretischen Betrachtung jenes unteilbare, in sich geschlossene Ganze, welches auch in der Kombination mit den verschiedenen Geisteskrankheiten seine Selbständigkeit bewahrt.

Noch leichter ergibt sich die Lösung der Frage nach der Verknüpfung der Hysterie mit Geistesstörungen für diejenigen, welche mit $Z$ i ehe $n$ in der Hysterie eine funktionelle Neurose sehen. In diesem Falle scheint eine solche Verbindung ohne jede Schwierigkeit stattfinden zu können, weil dann dieselbe nirgends hindernd im Wege steht. Das sich hier ergebende Verhältnis wäre dasselbe wie zwischen Neurasthenie und Geistesstörungen. Abgesehen davon, daß dieser Vergleich die Stellung zu den andersartigen Geisteskrankheiten zu beleuchten imstande ist, bietet er auch die Möglichkeit, die Entstehung der spezifisch hysterischen Psychosen ins Licht zu rücken. Denn wie man dann von neurasthenischen Geistesstörungen spricht, wenn Psychosen in ihrem Charakter nicht die Symptome anderer Geistes- 
störungen cindc utig erkennen lassen, sondern in ihrem Ablaufe die Merkmale der grundlegcnden Neurose bieten, so hat man auch erst dann das Recht, von hysterischer Psychose zu reden, wenn der Verlauf derselben ohne Schwierigkeit und ein. wandfrei die Symptome der im Körperlichen vorher nachgewiesenen und gleichsam ins Psychische übertragenen hysterischen Eigenart erkennen läßt. Alle anderen Fälle betrachte man als Kombinationen.

Eine dritte Auffassung nimmt der Hysterie im Verlaufe von Geistesstörungen ihre Selbständickeit, indem sie behauptet, das Vorkommen hysterischer Symptome bei letzteren sage blo $\beta$, da $\beta$ die vorhandenen Rindenerkrankungsprozesse in der gleichen Weise wie bei dem Rindenerkrankungsprozeß der Hysterie körperliche Erscheinungen machen können, welche den bei den letzteren auftretenden gleich sind. Die Eigenart der Hysterie wird also damit geleugnet, insoweit sie ihren Ausdruck in den hysterischen Stigmatis findet. Diese werden (nach Nissl) nur dadurch zu hysterischen Symptomen, daß sie auf dem Boden des hysterischen Zustandes entstehen, daß sic psychisch auslösbar und beeinflußbar sind und infolge des andaucrnd wirksamen hysterischen Zustandes jederzeit auftreten und ebenso spurlos wicder verschwinden können. Dieselben Stigmata können aber auch bei anderen Psychosen auftreten und gelten dann als der Effekt derselben allein, eine Kombination: Psychose plus Hysterie sci auszuschließen.

Der größte Teil der Fälle von Seelenstörungen mit ,sogenannten“" hysterischen Symptomen gehört im Sinne der letzterwähnten Auffassung zur Katatonie und Hebephrenie, der kleinere zum manisch-depressiven Irresein und zur Melancholie des Rückbildungsalters.

Mir scheint diese Auffassung ein circulus vitiosus zu sein, da sie ja die hysterischen Symptome aus dem hysterischen Zustande erkennen soll, zu dessen Kenntnis sie doch erst wieder aus ersteren gelangt.

Dieser Auffassung gegenüber hat man den Nachweis zu liefern, daß jene Geistesstörungen nicht imstande sind, die hysterischen Körpererscheinungen hervorzurufen: Das Wesen derselben erschöpft sich in den psychischen Äußerungen, jeder Affektausdruck ist bei denselben dem tatsächlich vorhandenen Affekte qualitativ adäquat. Kommt es darum zu körperlichen Erscheinungen, welche nicht mehr als der natürliche, aus dem menschlichen Typus abgeleitete Ausdruck der Affekte anzusehen sind, so muß für dieselben eine von der speziellen Geisteskrankbeit unabhängige kausale Begründung gefunden werden können. Dieselbe muß im Körperlichen gesucht werden, weil die Insuffizienz der Psyche aus der Natur der Sache gegeben ist. Man darf zur Bekräftigung dieser Ablehnung auch darauf hinweisen, daß im Falle der Richtigkeit der Theorie jene Fälle gewiß nicht, wie es tatsächlich der Fall ist, zu den Seltenheiten gehören würden, in denen neben den im einzelnen Falle spezifischen psychischen Symptomen abnorme körperliche Affektausdruckserscheinungen sich kundgeben.

Nissl glaubt, daß die Mcinungsverschiedenheiten über die Frage der Beziehungen zwischen Hysterie und den sogenannten einfachen Seelenstörungen von dem jeweiligen psychiatrischen Glaubensbekenntnis der einzelnen Autoren abhängen, Er meint damit die Auffassung der Geistesstörungen vom psychopathologischen Standpunkte, der methodisch seinen Ausdruck in der zweifachen Möglichkeit der symptomatologischen oder der klinischen Betrachtungsweise findet. Zwischen diesen beiden Betrachtungsweisen soll eine unüberbrückbare Kluft sich auftun. Wäre sie aber vielleicht doch überbrückbar, wenn man überlegte, daß für eine vollkommene Erfassung die eine mit der anderen sich verbinden muß? Das psychologische Gebiet verlangt eben mehr als jedes andere, daß man sich von einer Ausschließung 
irgendeines Gesichtspunktes bei der Betrachtung seines Vorwurfes fernhalte. Gerade die Hysterie ist geeignet, die Richtigkeit einer solchen Auffassung zu beweisen, zumal bei dieser Krankheit die klinische Betrachtungsweise allein zu keinem befriedigenden Resultate führt, vielmehr erst durch die Verbindung mit der symptomatologischen einen Einblick verschafft. Allerdings darf man die symptomatologische Betrachtungsweise nicht als der psychologischen inhaltlich gleich hinstellen. Sonst würde man aus ihr keine Schlüsse auf die hysterischen Krankheitszeichen ziehen können, weil die Natur derselben durch eine psychologische Betrachtung wenigstens nicht exschöpft, sondern nur durch eine psychophysiologische genügend erfaßt werden kann. Die klinisehe Betrachtungsweise soll bezüglich des Verhältnisses zwischen der Hysterie und den einfachen Seelenstörungen zur Erkenntnis führen, daß alle jene körperlichen Krankheitszeichen, welche man gewöhnlich als hysterische bezeichnet, von jeder einzelnen der früher erwähnten Geistestörungen hervorgebracht werden konnten, so daß es unnötig sei, in der Diagnose überhaupt noch die Verschicdenheit der Geistesstörung und der Hysterie zum Ausdruck zu bringen. Unrichtig erscheint mir, daß Nissl die Kombination der beiden Krankheiten mit der Einsetzung der Hysterie in die Ätiologie der bcstimmten Geisteskrankheit gleichsetzt. Wenigstens dürfte seine Ausdrucksweise keinen anderen Sinn zulassen: 1891 fände sich in der Heidelberger Klinik zum letzten Male die Diagnose: Hystero-Melancholie. „Von da tritt der ätiologische Faktor der Hysterie nur noch als hystcrisches Irresein diagnostisch zutage. “

Tatsächlich spielt nur bei diesem die Hysterie den ausschlaggebenden ätiologischen Faktor, während sie bei den anderen Geisteskrankheiten nur den Wert eines prädisponierenden Momentes hat. Als solches besagt sie eine Eigentümlichkeit des Nervensystems, welche wie alle neuropathischen Momente zugleich als prädisponierendes psychopathisches Moment gelten muß. Das hysterische Irresein hat daher notwendig die Symptome der Hysterie an sich, ist also durch die Veränderlichkeit und Flüchtigkeit des Zustandsbildes gekennzeichnet. Bei den anderen Geisteskrankheiten aber bilden die hysterischen Krankheitserscheinungen nur Zufälligkeiten, welche den Charakter und Ablauf des eigentlichen chronischen Krankheitsprozesses nicht tangieren, demselben nur eine Abänderung geben, durch welche sich der einzelne Fall von anderen der gleichen Krankheitsform abhebt.

Die hysterischen Psychosen sind in ihren Hauptsymptomen den gleichbenannten Psychosen nicht hysterischer Personen identisch. Thre Abtrennung von letzteren erfolgt auf Grund von Eigentümlichkeiten, welche als die Spiegelbilder der körperlichen Merkmale betrachtet werden müssen. Die volle Gewißheit über den Charakter einer Psychose pflegt man allerdings erst nach dem Auftreten und durch den Nachweis von Körperkrankheitserscheinungen zu bekommen; aber man kann sich durch häufige Beobachtung eine große Gewandtheit in der Beurteilung unterkommender Krankheitsfälle erwerben, wobei noch dazu einiges Zuwarten stets durch auftretende somatische Erscheinungen zum sicheren Urteile führt, wenn nicht schon ein vorhergegangener hysterischer Anfall die Diagnose beleuchtet hat. Der unmittelbare Ursprung aus einer 
Gemütserregung lenkt die Aufmerksamkeit des Diagnostizierenden nach der Richtung zur hysterischen Psychose.

Als charakteristische Merkmale der hysterischen Psychose betrachtet man allgemein :

1. Den plötzlichen Beginn und den plötzlichen Schluß des psychotischen Anfalles.

2. Die verhältnismäßig kurze Dauer (von selten mehr als einigen Wochen).

3. Die plötzliche vollständige Unorientiertheit über Ort, Zeit und Umgebung.

4. Die Lebhaftigkeit und Mannigfaltigkeit der Sinnestäuschungen und die romanhafte Verkettung der halluzinatorischen Erlebnisse.

5. Den phantastischen Inhalt der Halluzinationen.

6. Die Verwebung der realen Empfindungen in die halluzinatorischen Erlebnisse durch illusionäre Auslegungen.

7. Die nachfolgende partielle Amnesie.

8. Die (meist totale) Analgesie.

Die Diagnose des hysterischen Irreseins kann nie aus dem Ansehen des gerade vorliegenden Zustandsbildes gemacht werden, da in demselben keine Symptome vorkommen, welche dasselbe nach der erwähnten Seite charakterisieren und jede andere Grundkrankheit beweisend ausschließen. Auch wenn körperliche hysterische Erscheinungen vorhanden sind, können dieselben nicht als Beweise gelten, daß es sich beim gegebenen Zustandsbilde um hysterisches Irresein handle, am wenigsten demjenigen, der die Hysterie als funktionelle Neurose auffaßt, aber auch jenem nicht, der die Lehren der Salpêtrière verficht. Die von den meisten derselben veröffentlichten Krankheitsgeschichten reden zu deutlich eine entgegenlautende Meinung, als daß jemand hinter diesen Meistern Deckung finden könnte, wenn er sie zur Zeugenschaft für eine diese Annahme bejahende Stellungnahme aufrufen wollte. Es bleibt darum auch hier, wie ich das im besonderen für die stuporösen und Erregungszustandsbilder beim mechanisch-depressivem Irresein einerseits und bei der Katatonie andererseits auseinandersetzte, nichts anderes übrig, als aus der Zusammenfassung der über die ganze kranke Persönlichkeit bekannt gewordenen habituellen Züge und Eigenheiten, aller zeitlich auseinanderliegenden, für die Beurteilung des psychischen Wesens derselben in Betracht kommenden Vorfallenheiten sich ein Bild von der ätiologischen Abstammung des Zustandsbildes zu machen. Gelegentliche, aus anderweitiger Erfahrung bekannte Züge werden die - vielleicht sehr wahrscheinliche - Diagnose nahelegen, eine Gewißheit kann erst durch die eben erwähnte Erkenntnis gebracht werden. Die Schwierigkeit, Zustandsbilder des Stupors und der Erregung nach ihrer Ätiologie zu katalogisieren, erfährt also eine Weiterung über das katatone und 
das manisch-depressive Irresein hinaus, wenn man an das hysterische Irresein herankommt. Doch ist hier eine Entscheidung leichter zu treffen, da die Dauer eine kurze ist, während bei den genannten beiden Krankheiten neben der äußeren Ähnlichkeit des Zustandsbildes dessen Chronizität die Erkenntnis erschwert.

Wird die Entscheidung für das hysterische Irresein auf Grund vorliegender körperlicher Erscheinungen gefällt, dann könnte dieselbe nach Auffassung derjenigen, welche jede mit Hysterie verbundene Geisteskrankheit als hysterische bezeichnen, als richtig angesehen werden; auf jeden Fall wäre aber eine Entscheidung allein nach psychischen Symptomen der Beachtung unwert, weil, wie schon ausgeführt, solche Symptome ohne Begleitung typischer körperlicher Erscheinungen eine zu große Vieldeutigkeit der Auffassung zulassen, auch keines derselben als n ur für Hysterie kennzeichnend angesehen werden kann.

Auch in dieser Beziehung haben die gegenwärtigen Kriegserfahrungen Vorteil gebracht, indem sie die Richtigkeit der eben ausgeführten Anschauung in einer großen Anzahl der Fälle erkennen ließen. Wie oft mußte nach einiger Beobachtung eine im ersten Anhub gestellte und mit Zähigkeit verteidigte Ansicht geändert werden! Es gilt eben auch hier, daß es nur ein Gebot der Vorsicht ist, mit einem kategorischen Urteile so lange zurückzuhalten, bis die äußeren Verhältnisse und die Aufrollung der ganzen Persönlichkeit eine Klärung der Sachlage herbeiführen. Gerade in den Kriegsfällen haben wir leider nur zu oft die nötigen Anhaltspunkte nicht erlangt, weil wichtige Mitteilungen über das Vorleben der Kranken, den Beginn und ersten Anfang der Krankheit nicht nur in etwas zu kurzen Abbreviaturen gegeben, sondern großen Teiles an den Zwischenstationen unter den Übernahmsakten gebettet wurden. Gerade für Fälle, welche hysterische Symptome zeigten, sind solche Mitteilungen von außerordentlicher Wichtigkeit, weil die intellektuelle Potenz der Kranken nur an der Hand solcher Mitteilungen richtig beurteilt werden kann. So kann z. B. die Entscheidung, ob es sich um eine hysterische Psychose bei einem Imbezillen oder um eine Attacke einer Dementia praecox handle, kaum anders getroffen werden als unter Berücksichtigung solcher Mitteilungen. Daß weder der eine noch der andere Kranke zum Militärdienste tauglich sei, ist schließlich doch eine Sache, welche nur einen derzeit allerdings wichtigen, den Rahmen der medizinischen Beurteilung und Behandlung aber nicht umfassenden Faktor darstellt. Dem Arzte obliegt die weitere Aufgabe, sich über den Kranken ein detailliertes Bild zu schaffen und die Möglichkeit einer sozialen Tätigkeit und Betätigung prognostisch festzulegen.

Untersucht man viele Menschen, die mit den verschiedensten Krankheitszuständen behaftet sind - sagt Ganser ${ }^{1}$ ) — gleichmäßig nach

\footnotetext{
1) Archiv f. Psych. 1904. Zur Lehre vom hysterischen Dämmerzustande.
} 
allen Richtungen hin, so findet man ungemein häufig Störungen der Sensibilität und der Schleimhautreflexe, wie sie in ausgesprochenen Fällen der Hysterie als mehr oder weniger wichtige Elemente des Krankheitsbildes vorkommen und ebenso verhält es sich mit der für den hysterischen Charakter so bedeutungsvollen Suggestibilität. Nur bei einem Bruchteil der Fälle, die mit diesen Stigmatis behaftet sind, kommt es zur Entwicklung des hysterischen Irreseins-Bildes. Der Prozentsatz an hysterischem Irresein Kranker bleibt hinter dem der Hysteriker zurück, woraus man den Schluß ziehen kann und muß, daß, wenn man auch die Hysterie als eine weit verbreitete Krankheit ansehen darf, doch die Entstehung eines hysterischen Irreseins vom Vorhandensein und Eintritte bestimmter Umstände abhängt. Indem nun Nissl (fußend auf Kraepelin) die bei anderen Krankheitsformen als dem hysterischen Irresein vorkommenden hysterischen Erscheinungen als, wenn auch nicht im Wesen, so doch in der Genese verschiedene bezeichnete und zur Unterscheidung hysteriforme nannte, zog er damit den Kreis der hysterischen Psychosen an sich nicht enger, aber er nahm der Hysterie viel von ihrer Selbständigkeit. Wo es nicht gelingt, den hysterischen Charakter eines oder einer Kranken nachzuweisen, da können im Sinne Nissls Erscheinungen, welche man als hysterische aufzufassen nach wissenschaftlicher Gewohnheit berechtigt ist, nicht mehr als solche gelten. Denn: „Unter Hysterie versteht Krae pelin eine angeborene Krankheit, welche einen eigenartigen Zustand des Nervensystems bedingt, der klinisch dadurch zum Ausdruck gelangt, daß er zur Entwicklung des sogenannten hysterischen Charakters führt und sich andauernd insofern wirksam zeigt, daß jederzeit passagere körperliche Störungen und verschiedene Formen eines spezifischen Irreseins durch gefühlsstarke Vorstellungen hervorgerufen werden können." Vor allem sei darauf hingewiesen, daß diese Auffassung aus dem Jahre 1902 wenigstens nicht mehr in ihrer Gänze heute von Krae pelin festgehalten wird, da er in der letzten Auflage seines Lehrbuches (1915) nicht mehr nur gefühlsstarke Vorstellungen als die ätiologischen Faktoren der Hysterie bezeichnet, sondern psychische Zustände überhaupt.

Nissl folgert, daß nur dort von Hysterie gesprochen werden könne, wo der hysterische Charakter nachzuweisen sei. In den Worten Krae pelins scheint das nicht zu liegen, auch dürfte die Annahme sich nicht als richtig erweisen lassen, daß die Hysterie immer zur Entwicklung des hysterischen Charakters führe, so daß also für eine Kombination einer Psychose mit Hysterie der Nachweis des hysterischen Charakters keine unbedingte Notwendigkeit bildet. Außerdem widerspricht die Bezeichnung: angeborene Krankheit wohl zu offenkundig der Erfahrung, daß bei Personen, die seit Kindheit keinerlei Zeichen eines hysterischen Charakters hatten erkennen lassen, nach starken gemütlichen Erre- 
gungen, Unfällen und schmerzhaften Krankheiten der hysterische Charakter sich entwickelt, der dann die ganze übrige Lebenszeit oder für längere Zeit fortbestehn kann. Auch für diese Annahme kann ich auf meine gegenwärtigen Kriegserfahrungen mich berufen. Soweit die Erlangung von Berichten über den geistigen und nervösen Zustand unserer Patienten möglich war, wurden dieselben in das Kalkül der Krankheitsbeurteilung einbezogen. Bei Erkrankungen, die nach dem Beginne und dem Zustandsbilde die Vermutung nahelegten, daß sie dem hysterischen Irresein angehören, war es meist doppelt wichtig, solche Berichte zu erhalten. Da wurde es nun Tatsache, daß in den weitaus meisten Fällen während der militärfreien Zeit man an den jetzt kranken Individuen keine Auffälligkeiten bemerkt hatte, was schließlich um so schwerer in die Wagschale fällt, als gerade bezüglich der Krankheitszeichen der Hysterie eine so weitgehende und allgemeine Kenntnis auch in den Volkskreisen verbreitet ist, daß man es sicher nicht unterlassen hätte, darauf hinzuweisen. Wahrscheinlich hätte man jene Zeichen nicht als hysterische bezeichnet, aber unter den Auffälligkeiten, Charaktereigentümlichkeiten und epileptischen Anfällen genannt.

Wie bezüglich der Neurosen Hysterie und Neurasthenie, hat man auch von der hysterischen und neurasthenischen Psychose behauptet, daß dieselben voneinander wenigstens in vielen Fällen nicht zu unterscheiden seien. Wie man die ersteren nicht mit Sicherheit zu trennen vermag, wenn man allein den psychischen Habitus der betroffenen Person ins Auge faßt, und solange man nicht den Schwerpunkt der Unterscheidung in den körperlichen Erscheinungen der Hysterie festhält, so kann eine Scheidung der hysterischen Psychose von der neurasthenischen Psychose gleichfalls nur an der Hand des neurotischen Befundes gemacht werden. Bezüglich des rein psychischen Bildes wird eine Differenz in den $\mathrm{Zu}$ standsbildern kaum jemals festzustellen sein. Man muß den Standpunkt Möbius' vertreten, der die Neurasthenie als eine von der Hysterie gänzlich verschiedene Krankheit ansah und diese Verschiedenheit auch für die Beurteilung psychischer Störungen festhielt.

Möbius, welcher die Neurasthenie als eine von der Hysterie ganz verschiedene Krankheit ansah, erklärte für möglich, daß ein Hysterischer so gut wie irgendein anderer neurasthenisch sein könne. Mit dieser Annahme halte ich es verträglich, daß die hysterische Veränderung und die ihr entsprechenden spezifischen hysterischen Krankheitsmerkmale auf dem Boden der neurasthenischen Dauerermüdung zustande kommen. Denn der gemeinsame Untergrund bedingt noch lange keine Gleichheit der Ausdruckserscheinungen.

Die Dynamik der bei der Hysterie zum Ausdruck kommenden Nervenerregungen ist eine vom Gefühl abhängige, gleichzeitig von der 
Norm abweichende. Das Abweichen von der Norm besteht in einer abnormen Verteilung der Erregungsgrößen. Denn etwas ganz Neues gegenüber dem Verhalten des normalen Menschen bedeuten jene Erregungen nicht, dieselben treten ja auch vorübergehend bei diesem auf, geändert erscheint bei der Hysterie erstens ihre Zuweisung zu vollkommen inadäquat erscheinenden Reizen und zweitens das Mangelhafte in ihrer Lösung. Realisierung der hysterischen Erscheinungen ist undenkbar, wenn nicht Anknüpfungspunkte an wirkliches Erleben gegeben sind, da alles Krankhafte sich aus dem normalen Leben heraus entwickelt. Für diese Entwicklung ist dann allerdings der ,état hystérique" maßgebend, den man gar nicht als einen mentale zu bezeichnen braucht, da er doch in nichts anderem als in einem hohen Grade von Nervenlabilität einer bestimmten Spezifität besteht, die man in einer Tendenz zu heftigen Schwankungen der Nervendynamik finden wird. Die Auffassung der Hysterie als Änderung der Nervendynamik gegenüber der Norm ist geeignet, die Komplikation mancher Geistesstörungen durch hysterische Erscheinungen als leicht möglich zu erklären. Diese dynamischen Störungen sind imstande, auch alle Störungen im Assoziationsvorgange, wie dieselben in erster Linie das hysterische Irresein charakterisieren, zu erläutern.

Worin hat die erwähnte Änderung in der Nervendynamik ihren Grund? Darüber sind verschiedene Theorien aufgestellt, denen gemeinsam ist, daß jede nur ein einzelnes Symptom oder einzelne Symptome der Hysterie erklärt. Die Meynertsche Hypothese, daß es sich bei der Hysterie um Zirkulationsstörungen im Gebiete der Arteria chorioidea handle, erklärt eine eventuelle hysterische Hemianästhesie, während sie die Erklärung anderer Symptome nicht gibt. Knies spricht von einer vasomotorischen Erweiterung der Arterien, die eine Kompression des Sehnerven im Foramen opticum herbeiführe, eine Theorie, die man von dem Spezialfall der unilateralen Sehstörung auf andere hysterische Symptome nicht übertragen kann. Auch die Theorie Waltons von einer mechanischen Kommotion hat nicht für alle Fälle von Hysterie Geltung, sondern nur für die traumatische Hysterie. Eine allgemeine Zusammenfassung aller hysterischen Erscheinungen gibt die Theorie Férés, wonach die Erschöpfung entweder an sich die hysterischen Erscheinungen bedingt oder die infolge Erschöpfung auftretende verminderte Leistungsfähigkeit eine Vorstellung (z. B. der Lähmung) weckt, die dann die Lähmung bewirkt. Auch Löwenfeld betrachtet die Hysterie als Produkt der Hirnerschöpfung. Dieser Theorie gegenüber ist geltend gemacht worden, daß die eigentliche Erschöpfungsneurose die Neurasthenie darstelle.

Nach Grasset kämen alle hysterischen Symptome zustande durch Vereinigung oder Trennung des Zentrums für die höheren geistigen Funktionen (des 
präfrontalen Zentrums für Wille, Bewußtheit und Moral) von einem, zwei oder allen niederen Hirnzentren (die in Schläfen-, Hinterhaupts- und Scheitellappen gelegen und für Motilität, Sensibilität und Assoziationen bestimmend sein sollen).

Schtscherbak sucht die für Hysterie spezifische Störung in jenen Rinden. gebieten, in welchen Verknüpfungen zwischen den höheren psychischen und den höheren somatischen Zentren stattfinden. Das Wesen der Störung sucht er durch Amöboismus zu erklären, worunter er ein pathologisches Dendritenspiel versteht, das entweder eine pathologische Annäherung oder eine pathologische Trennung der Neurone bewirke. Durch erstere sollen motorische, vasomotorische und viscerale Erscheinungen, durch letztere Lähmungen und Anästhesien zustande kommen.

Ebensowenig wie über die Art und Ursache der Dynamikänderung ist man über den Zeitpunkt der Entstehung der damit verknüpften krankhaften Disposition des Nervensystems zu einer einheitlichen Ansicht gekommen. Obwohl einwandfreie Tatsachen für eine Erwerbung dieser Anlage durch Gifte, Traumen und Utberanstrengung sprechen, hält doch noch der größte Teil der Psychologen daran fest, daß dieselbe stets eine angeborene sei.

$\mathrm{Zu}$ einem sicheren Resultate kann man bezüglich des Ortes der hysterischen Veränderung gelangen. Theoretische Erwägungen, welche die Konsequenzen früherer Ausführungen über die den hysterischen Erscheinungen zugrunde liegenden psychischen Vorgänge sind, führen zum Ziele. Von den meisten Autoren ist diese Frage überhaupt nicht weiter behandelt worden, von den einen, weil die Lösung derselben gegeben war in ihrer Annahme, daß Vorstellungen die Genese der Hysterie beherrschen, von anderen, weil die Beeinflussung der Erscheinungen durch Vorstellungen eine andere Lösung als die der erstgenannten nicht zuzulassen schien und wieder anderen, weil sie alles Psychische in der Frage der Hysterie ausgeschaltet wissen wollten. Die ersten beiden Klassen von Autoren verlangten eine Lokalisation in der Hirnrinde, weil ohne eine solche weder das Zustandekommen noch die Lösung durch Vorstellungen erklärt werden könne; man statuierte also eine Theorie, welche ausgehend von der allgemeinen Annahme, daß die Hirnrindenzentren die Zentren der Vorstellungsbildung seien, von vornherein als selbstverständliche Zugabe zu den in Betracht gezogenen Hysterietheorien erscheinen mußte. Die dritte Ansicht entzog sich aber durch ihre Behauptung selbst jeder Einbeziehung in eine cerebrale Deutung.

In den früheren Darlegungen war die Feststellung gemacht worden, daß zum Zustandekommen von hysterischen Erscheinungen rein sensorielle Gefühle genügend seien unter ausdrücklichem Ausschlusse der höheren psychischen Vorgänge der Vorstellungsbildung. Die Zentren für jene Gefühle liegen subcortical. Also braucht man für die Hysterie als notwendigen ätiologischen Faktor für alle Fälle nur die subcorticalen Zentren. Die Schwierigkeit, welche aus den durch Vorstellungen verursachten hysterischen Krankheitserscheinungen gemacht wird, 
erledigt sich von selbst für jene, welche den für alle solche Erscheinungen gemeinsamen Angriffspunkt gemä $\beta$ der vorausgehenden Erkenntnis der psychologischen Genese in den rein nervösen und nicht unmittelbar mit dem Psychischen verknüpften Zentren des Gehirnes sehen. Anderen gegenüber, welche im entgegengesetzten Sinne die Frage lösen, gilt es, ihnen zuerst die Unrichtigkeit ihrer psychologischen Anschauungen nachzuweisen. Die früheren Ausführungen haben sich damit beschäftigt. Anschließend können dann die eben erst dargelegten Folgerungen geltend gemacht werden.

Allgemein nimmt man heute an, die notwendige Bedingung, da $\beta$ hysterische Symptome auftreten können, sei durch eine zeitlich dem Auftreten derselben vorangehende Disposition gegeben. Soll diese Annahme sinnvoll sein, dann kann sie nur besagen, daß jenes der Hysterie Vorangehende etwas von ihr qualitativ Unterschiedenes darstellen muß. Denn ein nur quantitativer Unterschied würde in dem Ausdrucke lediglich einen Intensititätsunterschied besagen, daß eine schwache Hysterie die Vorläuferin der stärkeren sei. Jedenfalls wäre auch sie bereits eine Hysterie, allerdings eine solche, welche bisher aus Mangel an objektiven Ausdruckserseheinungen noch nicht erkannt werden konnte. Die Festsetzung eines solchen Begriffes wäre zwecklos, weil sie doch nur ein theoretischer Rückschluß sein könnte, der unsere Kenntnisse um keinen Schritt vorwärts brächte und zur Vermehrung auch nichts beitragen könnte, weil er doch erst immer wieder aus der gewonnenen Kenntnis der eben erst als stärker bezeichneten Hysterie gebildet werden könnte.

Diese hysterische Disposition ist in einem anderen Sinne gedeutet von den Vertretern der psychischen Theorien als von jenen der psychophysischen Theorien. Für die ersteren erscheint sie als eine Tendenz der Psyche, im gegebenen Falle d.h. bei Erfüllung der nötigen Bedingungen sich in Form von abnormen Ausdruckserscheinungen zu offenbaren. Ob diese Tendenz in der Psyche selbst ihre Wurzel habe oder erst durch die Verbindung mit dem psychischen Anteile des menschlichen Wesens gewinnen könne, ist eine Frage, welche nur durch die rationale, nie durch die empirische Psychologie beantwortet werden kann. Zu ihrer Beantwortung muß von den Grundanschauungen über das Wesen der Psyche ausgegangen werden. Alle jene Anschauungen, welche neben der Wesensverschiedenheit auch eine gegenseitige Unbeeinflußbarkeit zwischen Psyche und Körper vertreten, begeben sich selbst der Möglichkeit, die körperlichen hysterischen Erscheinungen aus einer abnormen Tendenz der Psyche zu erklären. Denn wenn eine kausale Einwirkung überhaupt unmöglich ist, wie soll auf einmal eine abnorme Tendenz zuwege bringen, was die normale nicht kann? Wie sollte ein 
Defekt in der psychischen Reihe die Ursache zu einem solchen in der physischen werden? Für alle anderen Vertreter der genannten Richtung wäre die Entscheidung zu fällen nach ihrer Auffassung des Wesens der Psyche. Nachdem ich den psychophysischen Parallelismus ausgeschieden habe, frage ich jene, welche die Geistigkeit und Einfachheit der Psyche festhalten, wie sie mit dem einsinnigen Streben derselben eine solche abnorme und noch dazu nicht allgemein abnorme Zielstrebigkeit der Psyche vereinbaren wollen? Eine solche Zielstrebigkeit liefe der Natur der Psyche entgegen. Und wollte man auch von dieser Schwierigkeit absehen, dann bliebe die andere, wie die Psyche dazu komme, sich durch körperliche Erscheinungen zu äußern, welche von der Form der sonstigen Ausdrucksweise abweichen. Die Auffassung der Psyche als unmittelbarer Hervorbringungen der Gehirnrindentätigkeit hat mit den vorgenannten Auffassungen nichts gemein; die Lösung der Hysteriefrage wie jene der hysterischen Veranlagung stellt sie (in diesen Punkten) neben die psychophysischen Theorien. Ihr bedeutet ja der Name Psyche nichts weiter als den Gegensatz der Tätigkeit der Gehirnrindenzentren zu jener der anderen Gehirnzentren, also keinen Wesens-, sondern einen rein formellen Unterschied.

Die hysterische Disposition wird nach der psychischen Hysterietheorie durch einen (hysterischen) Geisteszustand, welcher lange vor dem Offenkundigwerden der Krankheit existiert, gebildet. Dieser Geisteszustand ist die causa prima et radix aller Hysteriesymptome, der geistigen ebenso wie der körperlichen. Es ist also die hysterische Veranlagung eine bestimmte Seelenbeschaffenheit, welche wesentlich in quantitativer Steigerung und eigenartiger Mischung gewisser allgemein menschlicher Charaktereigenschaften besteht (Gaupp).

Während von einer Reihe von Autoren an der Ererbtheit dieses Zustandes festgehalten wird, lassen andere eine Erwerbung desselben zu, tragen damit der Tatsachenlage Rechnung, weichen aber den Schwierigkeiten, welche gegen die Auffassung als solche geltend gemacht werden, nicht aus. Diese Schwierigkeiten erheben sich vor allem von seiten der Körpersymptome. Der Psyche müßte bei der hysterischen Anlage ein schöpferischer Einfluß auf die körperlichen Vorgänge zugeschrieben werden, durch den es ihr gelänge, im Laufe der Zeit die natürliche Anlage hauptsächlich der Nerven abzuändern, den ihr naturgemäß zukommenden Einfluß auf Unterbleiben oder Auftreten, Intensität und Extensität und Richtung in einen Einfluß auf die Qualität im Sinne eines kontradiktorischen Gegensatzes zu verwandeln. Das gilt vom psychischen Vermögen überhaupt. Deswegen ist es ganz gleichgültig, ob man die Zerstreutheit, die Lenksamkeit oder eine sonstige Eigentümlichkeit zur Erklärung heranzieht.

Auch das Affektleben bietet keine Eigentümlichkeiten, welche von 
vornherein als kennzeichnend für eine hysterische Grundlegung betrachtet werden könnten. Was man an demselben als für hysterische Disposition typisch bezeichnet hat, wurde nicht einmal von den eifrigsten Vertretern dieser Meinung als beweisend angesehen und stets erst dann als ,doch" hysterisch anerkannt, wenn andere schlagende Beweise dazugekommen waren. Tatsächlich ist es auch nicht immer die Kraft der Anschauung, welche ihr zu ihrer großen Verbreitung geholfen hat, sondern das Ansehen ihrer Wortführer.

Ja net hat Trübsinn und Traurigkeit als die vorherrschende Gemütsstimmung ebenso bei hysterischen Männern wie bei hysterischen Frauen bezeichnet, während andere das hysterische Weib als erregt, heiter, rührig, laut lachend, zu allen Torbeiten geneigt hinstellen, den hysterischen Mann aber als traurig, schlaff und trübsinnig. Doch liegt aus seinen Schriften kein Anhaltspunkt vor, in diesen Merkmalen, wie man es ihm unterstellt hat, die Grundlage zu suchen, welche eindeutig oder auch mit Sicherheit als die Vorbedingung einer Hysterie zu deuten wäre.

Hellpach hat als die der Hysteric zugrunde liegende seelische Eigenschaft die Lenksamkeit bezeichnet. Er beantwortet die selbstgestellte Frage nach dem, was nun die Lenksamkeit zur Hysterie fortentwickle, mit dem Hinweise auf eine in der Hysterie auftretende Isolierung der Persönlichkeit. Die Antwort scheint mir ebensowenig wie die angeschlossene Erläuterung dieses Ausdruckes als eine ,zu starke Nachinnenwendung der apperzeptiven Spannung" Klarheit zu bringen. Andererseits muß man auf jene Ausführungen des Autors verweisen, die von einem psychophysischen Riegel vor der Psychologie der Hysterie handeln. Nach denselben sollte man erwarten, daß er wenigstens neben der Lenksamkeit eine weitere physische Anlage in den nervösen Organen der Ausdrucksbewegungen annehme. Hellpach hat nirgends ausdrücklich davon gesprochen, auch nirgends eine Andeutung über diesen Punkt gemacht.

Die Vertreter der psychophysischen Theorie legen folgerichtig auch in der jetzt nächstberührten Frage das Hauptgewicht auf eine nervöse Disposition. Man spricht von Nervenschwäche und von Nervenerschöpfung; mir will scheinen: Synonyma. In beiden Fällen bezeichnet man mit diesem Ausdrucke die vorhandene Labilität des nervösen Gleichgewichtes, welche weder mit der hysterischen Disposition noch mit der Hysterie identifiziert werden darf. Dieselbe ist auch bei anderen Krankheiten vorhanden, vollständig unbestimmt in bezug auf ihren späteren Verlauf. Sie kann in sich begrenzt bleiben und es können sich Erscheinungen anschließen, die voll und ganz aus ihrem Rahmen heraus fallen. Dieses letztere ist bei der Hysterie der Fall. Welche inneren. Gründe für die Entscheidung maßgebend sind, liegt für uns außer aller Möglichkeit der Erkenntnis. Die Kenntnis der äußeren auslösenden Ursachen trägt dazu nichts bei, nicht einmal so weit, daß man bei Vorhandensein der gleichen äußeren Ursachen, welche bei einem nervösen Individuum einwirken, auch nur mit annähernder Gewißheit das Auftreten einer bestimmten Krankheitsform voraussagen könnte.

Die Vielfältigkeit der Möglichkeiten führte dazu, daß man theoretisch 
rückwärtsschauend jene Labilität mit einem speziellen Namen belegte, ohne daß es gelungen wäre, eine Charakteristicum auszuführen, welches sie nach einer bestimmten Seite kennzeichne und welches darum auch eine praktische Bewertung verlangte. Man muß sich damit bescheiden, jene nervöse Veranlagung als eine Krankheitsdiathese zu betrachten, welche die materielle Disposition zur Hysterie abgibt, wenn auch andere Momente als auslösende Faktoren, als unmittelbar auslösende Ursachen der Krankheit Hysterie an den Anfang der erkenntlichen Vorgänge zu stellen sind. Alles, was die Innervation übermäßig steigert, kann als geeignet angesehen werden, zur Hysterie zu disponieren. Aus dieser Veränderung der nervösen Funktionen kann man die psychischen Eigentümlichkeiten der Hysterie nach psychologischer Erfahrung ableiten. Das Umgekehrte ist eine Unmöglichkeit.

$\mathrm{Zu}$ wiederholten Malen schon habe ich darauf hingewiesen, daß man oft den sogenannten psychischen Stigmatis der Hysterie einen unzukömmlichen Erkenntniswert beilege. Damit sage ich nicht, daß dieselben zur allseitigen und umfassenden Klarstellung des Krankheitsbildes nicht beitragen können und tatsächlich beitragen, sondern nur, daß dieselben in der Loslösung von den sicher hysterischen sensiblen und motorischen Störungen das Krankheitsbild mehrdeutig lassen.

Als jene affektiven und intellektuellen Störungen, welche ohne Kenntnis des Krankheitsverlaufes und der körperlichen Krankheitsmerkmale die Diagnose Hysterie zu stellen gestatten sollen, bezeichnet man: 1. Die Beweglichkeit, nach Sydenham die Inkonstanz als das Konstanteste bei Hysterikern. 2. Die Neigung zu Widerspruch und Streit. 3. Den Geist der Doppelzüngigkeit, Lüge und Verstellung. Ist ein einzelnes dieser Merkmale oder sind vielleicht alle im gemeinsamen, gleichzeitigen Vorkommen spezifisch für Hysterie? Nein. Die gleichen Symptome werden bald vereinzelt, bald gehäuft bei allen degenerativen Geisteszuständen angetroffen, in ausgeprägter Art bei Hebephrenie und Schwachsinn (besonders dem sogenannten moralischen Schwachsinn). Man hat behauptet, daß die genannten Geistesanomalien durch die der Hysterie eigentümliche psychische Veränderung und die mit ihr verknüpfte Fernwirkung psychischer (besonders affektiver) Reize auf körperliche Innervationen ein bestimmtes Kolorit erhalten, aus dem sie als hysterische zu erkennen seien. Die Erfahrung hat die Bestätigung dieser Behauptung nicht gebracht. Denn es gibt eine nicht zu übersehende Zahl von Hysteriefällen, welchen die genannten Charaktereigentümlichkeiten abgehen. So habe ich wieder zur jetzigen Kriegszeit viele Fälle von sicherer Hysterie gesehen, an denen bei ausgesprochen 
hysterischen körperlichen Krankheitserscheinungen intellektuell-ethische Defekte nicht nachgewiesen werden konnten und bei welchen aus der Anamnese ihres Vorlebens keine Anhaltspunkte erkennen ließen, daß sie die ihnen durch Beruf und Familie auferlegten Pflichten nicht vollkommen erfüllt hätten.

Ich schließe mich der Anschauung Binswangers an, daß die degenerativen Charakteranomalien und die aus ihnen entspringende Handlungsweise der Kranken für sich allein keineswegs zur Aufstellung der Diagnose Hysterie genügen, da sie in ähnlicher Weise auch anderen degenerativen psychischen Krankheitszuständen eigentümlich sein können. Die übrigen, vorzugsweise aus den Stimmungsanomalien entspringenden Eigentümlichkeiten hysterischer Kranker finden sich in gleicher Weise auch bei der einfachen Neurasthenie. Bei dieser Sachlage wird man genötigt sein, die Diagnose Hysterie erst dann sichergestellt zu erachten, wenn außer diesen Charakteranomalien noch andere Kardinalsymptome der Hysterie in Form körperlicher Krankheitserscheinungen entweder zur Zeit der Beobachtung oder in der Vorgeschichte des Falles erkennbar auftreten oder aufgetreten sind.

Als ein für hysterische Disposition und Hysterie psychisches Symptom hat man die Suggestibilität der Hysteriker angeführt. Auch von ihr gilt, was von den psychischen Symptomen im allgemeinen bereits gesagt wurde: auch sie kann als ein Spezificum so lange nicht betrachtet werden, als sie nicht in körperlichen Erscheinungen ihren Ausdruck findet. Solange rein psychische Erscheinungen die Folge einer Suggestion sind, gelingt es auf keine Weise, irgendeine bestimmte Färbung in denselben aufzuzeigen, wodurch sie von den gleichen Zustandsbildern, wie sie gelegentlich bei Degenerierten, Imbezillen und schließlich auch Simulanten sich darbieten, sich unterscheiden.

Diese Eigenschaft der Suggestibilität hat viele Autoren dazu geführt, die Hysterie weder für eine Neurose allein, noch für eine Psychose allein, sondern für eine Psychose und Neurose zu erklären. Bei einer'großen Zahl von Hysteriefällen spielt in der Genese die Suggestion gar keine Rolle. Bei ihr kommt darum die Auffassung der Krankheit als einer Psychose nicht in Betracht, solange die ausgelösten Erscheinungen rein nervöser Natur sind. Damit fällt natürlich die Allgemeingültigkeit der Annahme, daß das Wesen der Hysterie neben dem Neurotischen etwas Psychotisches enthalte, schon gar - wie früher ausgeführt jener, welcher die Hysterie überhaupt den Stempel des Psychotischen. einfachhin aufdrücken will.

Es kann indessen nicht geleugnet werden, daß der Charakter der Hysterischen großenteils eine psychische Eigenart darbietet, welche; obwohl an sich nicht für ihn allein festliegend, doch in einer großen Zahl der Krankheitsfälle als Begleiterscheinung der sicher hysterischen 
körperlichen Krankheitserseheinungen erkennbar ist. Nachdem ich nur diese letzteren als die Wesenskennzeichen der Hysterie betrachte, komme ich zu dem Schlusse, daß jene Eigenart etwas Sekundäres, und zwar, weil nicht immer vorhanden, seiner Natur nach Sekundäres darstelle. Es ergibt sich vollständige Analogie mit der Sachlage bei Epileptikern. Auch bei diesen kommt es mit wenigen Ausnahmen zu einer Depravation des Charakters, welche im allgemeinen nach denselben Richtungen wie bei Hysterikern abläuft. Hier wie dort liegt die Ursache in jenen wiederkehrenden Störungen neurotischer Zufälligkeiten, welche mit Störungen der Perzeption und der Empfindungen, vor allem aber der mit den letzteren verbundenen Unlustgefühle verknüpft sind, auf welcher Grundlage dann mit psychologischer Notwendigkeit eine psychische Abnormalität sich ausbildet.

Eine große Zahl der Psychopathologen hält jenen sogenannten hysterischen Geisteszustand für das Primäre im Krankheitsbilde, sie hat sich gewöhnt, ihre Ansicht durch die Autorität der Meister der Salpêtrière zu stützen. Ich verweise ihnen gegenüber auf meine früheren Ausführungen über die Theorien der psychischen Genese der Hysterie, berufe mich gleichzeitig auf die Autorität Binswangers. Er weist in seiner Monographie die Umformulierung der Erfahrungserkenntnis, daß alle hysterischen Krankheitserscheinungen in unlösbaren Beziehungen zu Hirnrindenfunktionen stehen, in die Behauptung, da B alle hysterischen Krankheitserscheinungen psychischen Ursprunges seien und ausschließlich aus einer psychischen Störung erklärt werden können, als unhaltbar zurück. Sie widerspreche allgemein psychophysiologischen und klinischen Erwägungen. Die Erfahrung, daß auch jene Krankheitssymptome, welche bei ihrem ersten Auftreten einen Bewußtseinsvorgang als Wirkursache erkennen ließen, späterhin durch psychische Phänomene ausgelöst werden können, gibt keinen Beweis $f$ ür die erwähnte Ansicht. Auch nicht jene andere Erfahrung, daß bei vielen hysterischen Empfindungsstörungen von vornherein der Einfluß psychischer Vorgänge auf ihre Entstehung und ihre besondere Lokalisation deutlich zutage tritt.

Diese beiden Erfahrungstatsachen haben einen gemeinsamen Untergrund. Die in den Hirnzentren liegende Störung der Zentrentätigkeit ist - mit Ziehen gesprochen - eine geringfügige, so daß eben eine Beeinflussung durch die Psyche möglich ist. Vielleicht würde man nicht irregehen mit der Annahme, daß eben gerade die funktionelle Störung (im Gegensatz zu einer anatomischen) jenes Moment bildet, welches eine Beeinflußung von der psychischen Seite zuläßt. Die anatomische Fixation würde eine solche ausschließen, gerade in jenen Punkten, welche für die Hysterie als Hauptmerkmale bezeichnet werden: dem Wechsel und der Veränderlichkeit der hysterischen Krankheitser- 
scheinungen. Eine anatomische Festlegung müßte sich in typischen Assoziationsphänomenen oder in typisch gleichbleibenden neurotischen Erscheinungen äußern. Die entgegenlaufende Eigenart der Hysterie dürfte auch der Grund sein, daß die Schilderungen über die seelische Gesamtbeschaffenheit der Hysterischen, wie über den hysterischen Intellekt so oft und so weit auseinandergehen und daß die Diskussion: Hysterie Neurose oder Psychose? immer noch zu keinem endgültigen Abschlusse geführt hat. 